\title{
POSITIVE CONES AND GAUGES ON ALGEBRAS WITH INVOLUTION
}

\author{
VINCENT ASTIER AND THOMAS UNGER
}

\begin{abstract}
We extend the classical links between valuations and orderings on fields to Tignol-Wadsworth gauges and positive cones on finite-dimensional simple algebras with involution. We also study the compatibility of gauges and positive cones, and prove lifting results in the style of the Baer-Krull theorem for fields.
\end{abstract}

\section{INTRODUCTION}

The links between valuations and orderings on fields are well-known and are an essential tool in real algebra and in quadratic form theory, see for instance [5, 14, 19].

In the recent papers [22, 23] and the book [24], Tignol and Wadsworth introduced and started the development of the theory of "gauges", valuation-like functions on (finite-dimensional) semisimple algebras and central simple algebras with involution, while in the past few years we studied signatures of hermitian forms and their links to positivity [1, 2, 3] and used them to propose "positive cones", a notion of ordering for (finite-dimensional) simple algebras with involution, cf. [4].

It is therefore natural to wonder if there could be links between gauges and positive cones, and if so, if they would be similar to the classical ones in the field case between valuations and orderings. This paper presents a positive answer to both questions. We show that, on a finite-dimensional simple algebra $A$ with involution $\sigma$, the most natural construction of a "valuation ring" associated to a positive cone leads to a unique $\sigma$-special gauge (Section (5). We then show that a notion of compatibility between $\sigma$-invariant gauges and positive cones can be described by several equivalent conditions, reminiscent of the field case (Section 6). We proceed with a number of technical results about anisotropy of forms for use in the final section (Section 7) and conclude the paper by studying the lifting of positive cones from the residue algebra of a $\sigma$-invariant gauge, in the style of the Baer-Krull theorem for fields (Section 8).

2010 Mathematics Subject Classification. Primary 13J30,16W60; Secondary 16W10, 06F25, $16 \mathrm{~K} 20$.

Key words and phrases. Real algebra, algebras with involution, orderings, valuations. 


\section{NOTATION}

In this paper $F$ will always be a field of characteristic different from $2, A$ will always be an $F$-algebra, and $\sigma$ an $F$-linear involution. We will explicitly indicate when $A$ is finite-dimensional over $F$, simple or semisimple, and also when $F=\operatorname{Sym}(A, \sigma) \cap Z(A)$, where $\operatorname{Sym}(A, \sigma):=\{a \in A \mid \sigma(a)=a\}$ and $Z(A)$ denotes the centre of $A$.

For a subset $S \subseteq A$ we define $S^{\times}:=S \cap A^{\times}$, where $A^{\times}$is the set of invertible elements of $A$. For each $n \in \mathbb{N}$ we denote the involution $\left(a_{i j}\right)_{i, j} \mapsto\left(\sigma\left(a_{j i}\right)\right)_{i, j}$ on $M_{n}(A)$ by $\sigma^{t}$. For $a_{1}, \ldots, a_{\ell} \in \operatorname{Sym}(A, \sigma)$, we denote the hermitian form $A^{\ell} \times A^{\ell} \rightarrow A,(x, y) \mapsto \sigma\left(x_{1}\right) a_{1} y_{1}+\ldots+\sigma\left(x_{\ell}\right) a_{\ell} y_{\ell}$ by $\left\langle a_{1}, \ldots, a_{\ell}\right\rangle_{\sigma}$. For $a \in A^{\times}$, we denote the inner automorphism $A \rightarrow A, x \mapsto a x a^{-1}$ by $\operatorname{Int}(a)$. If $h$ is a hermitian form over $(A, \sigma)$, we denote by $D_{(A, \sigma)}(h)$ the set of elements of $A$ that are represented by $h$.

Let $X_{F}$ denote the space of orderings of $F$. We make the convention that orderings in $X_{F}$ always contain 0 . If $P \in X_{F}$, we denote by $F_{P}$ a real closure of $F$ at $P$.

For $P \in X_{F}$ and $k$ a subfield of $F$ we denote the convex closure of $k$ in $F$ with respect to $P,\left\{x \in F \mid \exists m \in k-m \leqslant_{P} x \leqslant_{P} m\right\}$, by $R_{k, P}$. By $v_{k, P}$ we denote the valuation on $F$ with valuation ring $R_{v_{k, P}}=R_{k, P}$. We also denote the unique maximal ideal of $R_{k, P}$ by $I_{k, P}$. Recall that $I_{k, P}=\left\{x \in F \mid \forall \varepsilon \in k^{\times} \cap P-\varepsilon \leqslant_{P}\right.$ $x \leqslant P \varepsilon\}$.

If $v$ is a valuation on $F$, we denote its value group $v\left(F^{\times}\right)$by $\Gamma_{v}$ and its residue field by $F_{v}$. In the context of graded rings later in the paper, we will also use the notation $F_{0}$ for $F_{v}$. Recall from [19, p. 72] that $v$ and $P$ are called compatible if for all $a, b \in F, 0<_{P} a \leqslant_{P} b$ implies $v(a) \geqslant v(b)$. See [19, Lemma 7.2] for equivalent characterizations; one that we will repeatedly use is $v(a+b)=\min \{v(a), v(b)\}$ for all $a, b \in P$. Also recall from [19, Thm. 7.21] that if $v$ is compatible with $P$, then $v=v_{k, P}$ for some subfield $k$ of $F$.

Several of our results use matrices with quaternion coefficients, cf. Zhang's paper [26], from which we recall what we need in Appendix A. Throughout the paper we make the convention that eigenvalue means right eigenvalue.

\section{GAuges}

Gauges were defined as a general notion of "valuation map" on finite-dimensional semisimple $F$-algebras by Tignol and Wadsworth, cf. [22, 23, 24] from which we recall the main definitions below. We assume for the remainder of this section that $A$ is a finite-dimensional semisimple $F$-algebra.

Definition 3.1. Let $v: F \rightarrow \Gamma_{v} \cup\{\infty\}$ be a valuation on $F$ and let $\Gamma$ be a totally ordered abelian group, containing $\Gamma_{v}$. A map $w: A \rightarrow \Gamma \cup\{\infty\}$ is

(1) a $v$-value function on $A$ if for all $x, y \in A$ and $\lambda \in F$, we have

$$
w(x)=\infty \Leftrightarrow x=0 ; w(x+y) \geqslant \min \{w(x), w(y)\} ; w(\lambda x)=v(\lambda)+w(x) ;
$$


(2) surmultiplicative if $w(1)=0$ and $w(x y) \geqslant w(x)+w(y)$, for all $x, y \in A$;

(3) a $v$-norm if $A$ has a splitting basis, i.e., an $F$-basis $\left\{e_{1}, \ldots, e_{m}\right\}$ such that

$$
w\left(\sum_{i=1}^{m} \lambda_{i} e_{i}\right)=\min _{1 \leqslant i \leqslant m}\left(v\left(\lambda_{i}\right)+w\left(e_{i}\right)\right), \quad \forall \lambda_{1}, \ldots, \lambda_{m} \in F .
$$

Let $w$ be a surmultiplicative $v$-value function on $A$ and let $\gamma \in \Gamma$. We consider the abelian groups

$$
A_{\geqslant \gamma}:=\{x \in A \mid w(x) \geqslant \gamma\}, A_{>\gamma}:=\{x \in A \mid w(x)>\gamma\} \text {, and } A_{\gamma}:=A_{\geqslant \gamma} / A_{>\gamma}
$$

and define

$$
\operatorname{gr}_{w}(A):=\bigoplus_{\gamma \in \Gamma} A_{\gamma},
$$

which is a graded $\operatorname{gr}_{v}(F)$-algebra. Recall that a homogeneous 2-sided ideal $I$ of $\operatorname{gr}_{w}(A)$ is a 2-sided ideal of $\operatorname{gr}_{w}(A)$ such that $I=\bigoplus_{\gamma \in \Gamma} I_{\gamma}$ where $I_{\gamma}:=A_{\gamma} \cap I$ and that $\operatorname{gr}_{w}(A)$ is called (graded) semisimple if it does not contain any nonzero homogeneous two-sided nilpotent ideal.

If $w$ is a surmultiplicative $v$-value function on $A$, we write

$$
R_{w}:=\{a \in A \mid w(a) \geqslant 0\} \quad \text { and } \quad I_{w}:=\{a \in A \mid w(a)>0\}
$$

for $A_{\geqslant 0}$ and $A_{>0}$, respectively.

We also define $\Gamma_{w}:=w(A \backslash\{0\})$ and note that it is a union of cosets of $\Gamma_{v}$, and is not a group in general.

Definition 3.2. Let $v: F \rightarrow \Gamma_{v} \cup\{\infty\}$ be a valuation on $F$ and let $\Gamma$ be a totally ordered abelian group, containing $\Gamma_{v}$. A map $w: A \rightarrow \Gamma \cup\{\infty\}$ is a $v$-gauge on $A$ if it is a surmultiplicative $v$-value function that is a $v$-norm and such that $\operatorname{gr}_{w}(A)$ is a semisimple $\operatorname{gr}_{v}(F)$-algebra.

If $\sigma$ is an $F$-linear involution on $A$, then a gauge (and more generally a $v$-value function) $w$ on $A$ is called $\sigma$-invariant if $w \circ \sigma=w$, and $\sigma$-special if $w(\sigma(x) x)=$ $2 w(x)$ for all $x \in A$. (Note that [23, Def. 1.2] has the additional assumption that $A$ is simple.)

Remark 3.3. The existence of a $\sigma$-special surmultiplicative $v$-value function on $A$ implies that $\sigma$ is anisotropic (i.e., $\sigma(x) x=0$ implies $x=0$ for every $x \in A$ ), as observed in the first line of the proof of [23, Prop. 1.1]. Also, a $\sigma$-special surmultiplicative $v$-value function is $\sigma$-invariant, cf. [23, Prop. 1.1].

Remark 3.4. Note that if $w$ is a $v$-gauge on $A$, then $A_{0}=R_{w} / I_{w}$ is a finitedimensional semisimple $F_{v}$-algebra which is not simple in general, cf. [22, Prop. 2.1. and $\S f f$.$] . It follows that I_{w}$ is the Jacobson radical of $R_{w}$, cf. [7, p. 1685].

If $w$ is a $\sigma$-invariant surmultiplicative $v$-value function, $\sigma$ induces an involution on $A_{0}$, which we denote by $\sigma_{0}$, and, if $\sigma_{0}$ is anisotropic, [23, Prop. 1.1 (b) $\Rightarrow(\mathrm{a})$ ] shows that $w$ is $\sigma$-special.

For future use we record the following two results. 
Lemma 3.5. Let $w$ be a $\sigma$-special $v$-value function on $A$, where $v$ is any valuation on $F$. Let $a \in \operatorname{Sym}(A, \sigma)$ and $r \in \mathbb{N}$. Then $w(a)>\alpha$ if and only if $w\left(a^{2^{r}}\right)>2^{r} \alpha$.

Proof. By induction on $r$. Since $w\left(a^{2^{r+1}}\right)=w\left(\sigma\left(a^{2^{r}}\right) a^{2^{r}}\right)=2 w\left(a^{2^{r}}\right)$, we have $w\left(a^{2^{r+1}}\right)>2^{r+1} \alpha$ if and only if $w\left(a^{2^{r}}\right)>2^{r} \alpha$.

Proposition 3.6. Let $w$ be a $\sigma$-special surmultiplicative $v$-value function on $A$, where $v$ is any valuation on $F$. Then $\operatorname{gr}_{w}(A)$ is semisimple.

Proof. Since $w$ is surmultiplicative and $\sigma$-special, we have $w \circ \sigma=w$, cf. Remark 3.3. Thus $\sigma$ induces a grade-preserving involution $\widetilde{\sigma}$ on $\operatorname{gr}_{w}(A)$. Let $I$ be a homogeneous nilpotent 2-sided ideal of $\operatorname{gr}_{w}(A)$, and let $a \in I_{\alpha}$ for some $\alpha \in \Gamma$, so $a=b+A_{>\alpha}$ for some $b \in A_{\geqslant \alpha}$. If $w(b)>\alpha$ then $a=0$. If $w(b)=\alpha$ then $w(\sigma(b) b)=2 \alpha$ and $\widetilde{\sigma}(a) a=\sigma(b) b+A_{>2 \alpha}$ in $A_{2 \alpha}=A_{\geqslant 2 \alpha} / A_{>2 \alpha}$ (by definition of the product in the graded ring, cf. [24, p. 98]). Since $I$ is a 2-sided ideal, $\widetilde{\sigma}(a) a \in I$ and thus there is $r \in \mathbb{N}$ such that $(\widetilde{\sigma}(a) a)^{2^{r}}=0$. Therefore, $(\sigma(b) b)^{2^{r}}+A_{>2 \cdot 2^{r} \alpha}=0$ in $A_{2 \cdot 2^{r} \alpha}$, i.e., $w\left((\sigma(b) b)^{2^{r}}\right)>2 \cdot 2^{r} \alpha$. By Lemma 3.5 we get $w(\sigma(b) b)>2 \alpha$, so $w(b)>\alpha$ and thus $a=0$.

Remark 3.7. Let $v$ be a valuation on $F$ such that $\operatorname{char} F_{v}=0$, and let $B$ be a finite-dimensional semisimple $F$-algebra. Then every $v$-gauge on $B$ is tame, cf. [22, Def. 1.5, Cor. 3.6].

Finally, we recall a special case of [23, Thm. 6.1]:

Proposition 3.8. Let $B$ be a finite-dimensional simple $F$-algebra with $F$-linear involution $\tau$ such that $F=\operatorname{Sym}(B, \tau) \cap Z(B)$, and let $v$ be a valuation on $F$ such that char $F_{v}=0$ and with Henselization $\left(F^{h}, v^{h}\right)$. Then:

(1) If $\tau \otimes \mathrm{id}_{F^{h}}$ is anisotropic then there is a $\tau$-special v-gauge on $B$.

(2) Let $y$ be a $\tau$-special v-gauge on $B$. Then $y$ is the unique $\tau$-invariant v-gauge on $B$ and is tame.

Proof. Note that $\tau \otimes \mathrm{id}_{F^{h}}$ is also anisotropic in (2), as observed at the start of the proof of [23, Thm. 6.1], so that $\tau \otimes \mathrm{id}_{F^{h}}$ is anisotropic in (1) and (2). It follows that the centre $L$ of the $F^{h}$-algebra $B \otimes_{F} F^{h}$ must be a field, see the third paragraph of the proof of [23, Thm. 6.1]. Since char $F_{v}=0$, every algebraic extension of $L$ is tamely ramified (cf. [24, Def. A.4]), so that the maximal tamely ramified extension of $L$ is its algebraic closure, and splits $B \otimes_{F} F^{h}$. Since furthermore $L$ is tame over $F^{h}, B \otimes_{F} F^{h}$ is tame over $F^{h}$ (cf. [23, Start of §2]), and so the hypothesis of [23, Thm. 6.1] is satisfied.

\section{Positive cones}

In [4] we defined positive cones on finite-dimensional simple $F$-algebras $A$ with involution $\sigma$, where $F=Z(A) \cap \operatorname{Sym}(A, \sigma)$. (Note that such algebras were simply called " $F$-algebras with involution" in [4].) More general algebras, specifically finite-dimensional semisimple $F$-algebras $A$ with involution $\sigma$ where $F \subseteq Z(A) \cap$ 
$\operatorname{Sym}(A, \sigma)$, will naturally appear as residue algebras of gauges, and motivate the following relaxation of the hypotheses on $A$ and $\sigma$ :

Definition 4.1. Let $F$ be a field and $A$ an $F$-algebra. Let $\sigma$ be an $F$-linear involution on $A$. Let $P \in X_{F}$. A prepositive cone $\mathscr{P}$ on $(A, \sigma)$ over $P$ is a subset $\mathscr{P}$ of $\operatorname{Sym}(A, \sigma)$ such that

(P1) $0 \in \mathscr{P}$;

$(\mathrm{P} 2) \mathscr{P}+\mathscr{P} \subseteq \mathscr{P}$

(P3) $\sigma(a) \cdot \mathscr{P} \cdot a \subseteq \mathscr{P}$ for all $a \in A$;

(P4) $\mathscr{P}_{F}:=\{u \in F \mid u \mathscr{P} \subseteq \mathscr{P}\}$ is equal to $P$ (we say that $P$ is the ordering associated to $\mathscr{P})$;

(P5) $\mathscr{P} \cap-\mathscr{P}=\{0\}$ (we say that $\mathscr{P}$ is proper).

A prepositive cone that is maximal with respect to inclusion is called a positive cone.

We define $\operatorname{Nil}[A, \sigma]$ to be the set of all $P \in X_{F}$ such that there is no positive cone on $(A, \sigma)$ over $P$, cf. [4, $\S 2$ and Prop. 6.6]. Note that $\operatorname{Nil}[A, \sigma]$ only depends on the Brauer class of $A$ and the type of $\sigma$.

Note that a prepositive cone $\mathscr{P}$ induces a partial ordering $\leqslant \mathscr{P}$ on $A$ defined by $a \leqslant \mathscr{P} b$ if and only if $b-a \in \mathscr{P}$. Moreover, since $\mathscr{P}$ is closed under sums, we have $a \leqslant \mathscr{P} b$ and $c \leqslant \mathscr{P} d$ implies $a+c \leqslant \mathscr{P} b+d$. As usual, we write $a<\mathscr{P} b$ for $a \leqslant \mathscr{P} b$ and $a \neq b$. Also note that $\mathscr{P} \neq\{0\}$ by $(\mathrm{P} 4)$.

Remark 4.2. Let $\mathscr{P} \subseteq A, \mathscr{P} \neq\{0\}$ and suppose $\mathscr{P}$ satisfies (P5), then to prove that $\mathscr{P}_{F}=P$ for a given $P \in X_{F}$, it suffices to prove that $P \subseteq \mathscr{P}_{F}$.

Example 4.3. Let $E$ be one of $F, F(\sqrt{-1})$ or $(-1,-1)_{F}$ (the Hamilton quaternion division algebra over $F$ ) and let - denote the identity on $F$ or conjugation in the remaining cases. Let $P \in X_{F}$. Recall that $M \in \operatorname{Sym}\left(M_{n}(E),{ }^{-t}\right)$ is positive semidefinite with respect to $P$ if and only if $\bar{x}^{t} M x \in P$ for all $x \in E^{n}$ (see Appen$\operatorname{dix} \mathrm{A}$ for the quaternionic case). The only two (pre)positive cones on $(E,-)$ over $P$ are $P$ and $-P$, since $\operatorname{Sym}(E,-)=F$. Therefore, by [4, Prop. 4.10], the only two (pre)positive cones on $\left(M_{n}(E),{ }^{t}\right)$ are the set $\operatorname{PSD}_{n}(E, P)$ of positive semidefinite matrices with respect to $P$ and the set of negative semidefinite matrices with respect to $P$.

Lemma 4.4. Let $A$ be an F-algebra with F-linear involution $\sigma$. Let $\mathscr{P}$ be a prepositive cone on $(A, \sigma)$ over $P \in X_{F}$, and let $L$ be any subfield of $Z(A) \cap$ $\operatorname{Sym}(A, \sigma)$ containing $F$. Then $P$ extends to $L$.

Proof. Let

$$
\Sigma:=\left\{\sum_{i=1}^{n} \ell_{i}^{2} p_{i} \mid n \in \mathbb{N}, \ell_{i} \in L, p_{i} \in P\right\} .
$$

Then $\Sigma$ is closed under sums and products (since $P$ is), and contains $L^{2}$. If $\Sigma \cap-\Sigma=\{0\}$ then $\Sigma$ is a preordering on $L$ containing $P$, which proves the result. 
Assume $\Sigma \cap-\Sigma \neq\{0\}$. Then there are $n \in \mathbb{N}, \ell_{i} \in L$ and $p_{i} \in P$ such that $\sum_{i=1}^{n} \ell_{i}^{2} p_{i}=0$ where not all $\ell_{i}^{2} p_{i}$ are equal to 0 . For instance, $\ell_{1}^{2} p_{1} \neq 0$. Let $a \in \mathscr{P} \backslash\{0\}$. Then

$$
0=\left(\sum_{i=1}^{n} \ell_{i}^{2} p_{i}\right) a=\sum_{i=1}^{n} p_{i} \sigma\left(\ell_{i}\right) a \ell_{i} .
$$

But $p_{i} \sigma\left(\ell_{i}\right) a \ell_{i} \in \mathscr{P}$ for every $i$, since $a \in \mathscr{P}$, and

$$
-p_{1} \sigma\left(\ell_{1}\right) a \ell_{1}=\sum_{i=2}^{n} \ell_{i}^{2} p_{i} a,
$$

with $p_{1} \sigma\left(\ell_{1}\right) a \ell_{1} \neq 0$. So we have a nonzero element in $\mathscr{P} \cap-\mathscr{P}$, contradicting (P5).

Lemma 4.5. Let $A$ be a finite-dimensional simple $F$-algebra with $F=\operatorname{Sym}(A, \sigma) \cap$ $Z(A)$. Let $\mathscr{P}$ be a prepositive cone on $(A, \sigma)$ over $P \in X_{F}$ and let $L \subseteq F_{P}$ be a field extension of $F$. Then $A \otimes_{F} L$ is simple and $L=\operatorname{Sym}\left(A \otimes_{F} L, \sigma \otimes \mathrm{id}\right) \cap Z\left(A \otimes_{F} L\right)$.

Proof. By [3, Prop. 6.6] we have $P \in \widetilde{X}_{F}=X_{F} \backslash \mathrm{Nil}[A, \sigma]$ and by [3, Rem. 6.2] we have that $A \otimes_{F} F_{P}$ is simple. It follows that $A \otimes_{F} L$ is simple.

Clearly $L \subseteq \operatorname{Sym}\left(A \otimes_{F} L, \sigma \otimes \mathrm{id}\right) \cap Z\left(A \otimes_{F} L\right)$. We consider two cases:

If $F=Z(A)$ then $F \otimes_{F} L=Z(A) \otimes_{F} L$ i.e., $L=Z\left(A \otimes_{F} L\right)$ (cf. [21, Chap. 8, Thm. 3.2(i)]), and the result follows.

If $F \varsubsetneqq Z(A)$. Then $[Z(A): F]=2$ and thus $\left[Z\left(A \otimes_{F} L\right): L\right]=2$. In case $Z\left(A \otimes_{F} L\right) \not \subset \operatorname{Sym}\left(A \otimes_{F} L, \sigma \otimes \mathrm{id}\right)$ then $\left[Z\left(A \otimes_{F} L\right): \operatorname{Sym}\left(A \otimes_{F} L, \sigma \otimes \mathrm{id}\right) \cap Z\left(A \otimes_{F} L\right)\right]$ is also 2 , and thus $L=\operatorname{Sym}\left(A \otimes_{F} L, \sigma \otimes \mathrm{id}\right) \cap Z\left(A \otimes_{F} L\right)$. In case $Z\left(A \otimes_{F} L\right) \subseteq$ $\operatorname{Sym}\left(A \otimes_{F} L, \sigma \otimes \mathrm{id}\right)$ then for every $a \in Z(A)$ we have $a \otimes 1 \in Z\left(A \otimes_{F} L\right)$ and thus $\sigma(a) \otimes 1=a \otimes 1$, from which it follows that $\sigma(a)=a$ and thus $Z(A) \subseteq \operatorname{Sym}(A, \sigma)$, a contradiction to $F \neq Z(A)$.

Proposition 4.6. Let $A$ be a finite-dimensional F-algebra with F-linear involution $\sigma$. Let $\mathscr{P}$ be a prepositive cone on $(A, \sigma)$ over $P \in X_{F}$ and let $(L, Q)$ be an ordered field extension of $(F, P)$. Then $\mathscr{P} \otimes 1:=\{a \otimes 1 \mid a \in \mathscr{P}\}$ is contained in a positive cone $\mathscr{P}^{\prime}$ on $\left(A \otimes_{F} L, \sigma \otimes \mathrm{id}\right)$ over $Q$.

If $\mathscr{P}$ is a positive cone, then $\mathscr{P}=\mathscr{P}^{\prime} \cap A$ (where we identify $\mathscr{P}$ with $\mathscr{P} \otimes 1$ and $A$ with $A \otimes 1)$; furthermore, if $A$ is simple and $F=\operatorname{Sym}(A, \sigma) \cap Z(A)$, then $\mathscr{P}^{\prime}$ is unique.

Proof. Observe that the results in [4, §5.2] hold when $A$ is a finite-dimensional $F$ algebra with $F$-linear involution $\sigma$, and where Definition 4.1 is used for prepositive cones. Then the first statement is [4, Prop. 5.8].

Since $\mathscr{P}^{\prime} \cap A$ is easily checked to be a prepositive cone on $(A, \sigma)$ over $F$, the second statement is clear since $\mathscr{P}$ is then a positive cone included in $\mathscr{P}^{\prime} \cap A$.

The final statement follows from Lemma 4.5] and [4, Thm. 7.5].

We can now relate positive cones over $F$ as defined in this paper and positive cones over $Z(A) \cap \operatorname{Sym}(A, \sigma)$ as defined in [4] for $A$ finite-dimensional and simple: 
Proposition 4.7. Assume that $A$ is a finite-dimensional simple $F$-algebra with $F$ linear involution $\sigma$. Let $\mathscr{P} \subseteq \operatorname{Sym}(A, \sigma), P \in X_{F}$, and $F_{\sigma}:=\operatorname{Sym}(A, \sigma) \cap Z(A)$. The following are equivalent:

(1) $\mathscr{P}$ is a positive cone on $(A, \sigma)$ over $P$.

(2) $\mathscr{P}$ is a positive cone on $(A, \sigma)$ over some ordering on $F_{\sigma}$ that extends $P$.

Proof. $(1) \Rightarrow(2)$ : We only have to check that $\mathscr{P}_{F_{\sigma}}$ is an ordering on $F_{\sigma}$. By Proposition 4.6, $\mathscr{P}=\mathscr{P}^{\prime} \cap A$ with $\mathscr{P}^{\prime}$ a positive cone on $\left(A \otimes_{F} F_{P}, \sigma \otimes \mathrm{id}\right)$, and since $P$ extends to $F_{\sigma}$ (by Lemma 4.4) we can assume that $F_{\sigma} \subseteq F_{P}$. The set $\mathscr{P}_{F_{\sigma}}$ is clearly a preordering on $F_{\sigma}$, so it remains to show that $\mathscr{P}_{F_{\sigma}} \cup-\mathscr{P}_{F_{\sigma}}=F_{\sigma}$. Let $u \in F_{\sigma} \subseteq F_{P}=\left(\mathscr{P}^{\prime}\right)_{F_{P}} \cup-\left(\mathscr{P}^{\prime}\right)_{F_{P}}$. If $u \in\left(\mathscr{P}^{\prime}\right)_{F_{P}}$ then, for every $a \in \mathscr{P}$, $u a \in \mathscr{P}^{\prime} \cap A=\mathscr{P}$, so $u \in \mathscr{P}_{F_{\sigma}}$. Similarly, if $u \in-\left(\mathscr{P}^{\prime}\right)_{F_{P}}$ we obtain $u \in-\mathscr{P}_{F_{\sigma}}$, which proves the result.

$(2) \Rightarrow(1)$ : We only have to check property $(\mathrm{P} 4)$. It is clear that $\mathscr{P}_{F}$ is a preordering on $F$, and we only have to check that $\mathscr{P}_{F} \cup-\mathscr{P}_{F}=F$. Let $u \in$ $F \subseteq F_{\sigma}$. If $u \in \mathscr{P}_{F_{\sigma}}$ then $u \mathscr{P} \subseteq \mathscr{P}$, so $u \in \mathscr{P}_{F}$. Similarly, if $u \in-\mathscr{P}_{F_{\sigma}}$ then $u \in-\mathscr{P}_{F}$, proving the result.

Definition 4.8. For a subset $S$ of $A$ and $P$ in $X_{F}$, we define

$$
\mathscr{C}_{P}(S):=\left\{\sum_{i=1}^{n} u_{i} \sigma\left(x_{i}\right) s_{i} x_{i} \mid n \in \mathbb{N}, u_{i} \in P, x_{i} \in A, s_{i} \in S\right\},
$$

and

$$
\mathscr{C}(S):=\left\{\sum_{i=1}^{n} \sigma\left(x_{i}\right) s_{i} x_{i} \mid n \in \mathbb{N}, x_{i} \in A, s_{i} \in S\right\} .
$$

4.1. Positive cones on finite-dimensional simple algebras with involution. In this section let $A$ be a finite-dimensional simple $F$-algebra with $F$-linear involution $\sigma$ such that $F=\operatorname{Sym}(A, \sigma) \cap Z(A)$ and let $\mathscr{P}$ be a positive cone on $(A, \sigma)$ over $P \in X_{F}$.

Lemma 4.9. Let $\mathscr{P}^{\times}:=\mathscr{P} \cap A^{\times}$. Then $\mathscr{P}=\mathscr{C}\left(\mathscr{P}^{\times}\right)$.

Proof. By [4, Def. 3.11 and Thm. 7.5], $\mathscr{P}=\mathscr{C}_{P}(S)$ for some set $S$ of invertible elements in $A$. Therefore, $\mathscr{P}$ is contained in $\mathscr{C}_{P}\left(\mathscr{P}^{\times}\right)$, and contains it by (P2), (P3) and (P4), so that $\mathscr{P}=\mathscr{C}_{P}\left(\mathscr{P}^{\times}\right)$.

But, for $u \in P \backslash\{0\}, x \in A$ and $b \in \mathscr{P}^{\times}$we have

$$
u \sigma(x) b x=\sigma(x)(u b) x, \text { with } u b \in \mathscr{P}^{\times},
$$

so that $\mathscr{C}_{P}\left(\mathscr{P}^{\times}\right)=\mathscr{C}\left(\mathscr{P}^{\times}\right)$.

Recall that the involution $\sigma$ is called positive at $P$ if the form $\operatorname{Trd}_{A}(\sigma(x) x)$ is positive definite at $P$, cf. [4, §6].

Proposition 4.10. The involution $\sigma$ is positive at $P$ if and only if $\left(A \otimes_{F} F_{P}, \sigma \otimes\right.$ $\mathrm{id}) \cong\left(M_{n_{P}}\left(D_{P}\right),^{-t}\right)$, where $n_{P} \in \mathbb{N}, D_{P} \in\left\{F_{P}, F_{P}(\sqrt{-1}),(-1,-1)_{F_{P}}\right\}$, and denotes the identity on $F_{P}$ and conjugation in the remaining cases. 
Proof. By [3, Rem. 4.7], $\sigma$ is positive at $P$ if and only if $\left(A \otimes_{F} F_{P}, \sigma \otimes\right.$ id $) \cong$ $\left(M_{n_{P}}\left(D_{P}\right), \operatorname{Int}\left(\Phi_{P}\right) \circ^{-t}\right)$, where $\Phi_{P}$ is a positive definite matrix over $D_{P}$. This matrix has a square root $\sqrt{\Phi_{P}}$, and applying $\operatorname{Int}\left({\sqrt{\Phi_{P}}}^{-1}\right)$ gives the desired isomorphism.

Remark 4.11.

(1) Recall that if $1 \in \mathscr{P}$, then the involution $\sigma$ is positive at $P$, cf. [4, Cor. 7.7].

(2) By Proposition 4.10, if $\sigma$ is positive at $P$ there exist extensions $L$ of $F$ (even finite ones), with $L \subseteq F_{P}$ such that $\left(A \otimes_{F} L, \sigma \otimes \mathrm{id}\right) \cong\left(M_{n_{P}}\left(E_{L}\right),{ }^{-t}\right)$, where $E_{L} \in\left\{L, L(\sqrt{-1}),(-1,-1)_{L}\right\}$. For any such extension $L$ we fix an isomorphism $f_{L}:\left(A \otimes_{F} L, \sigma \otimes \mathrm{id}\right) \stackrel{\sim}{\longrightarrow}\left(M_{n_{P}}\left(E_{L}\right),{ }^{t}\right)$ of $L$-algebras with involution.

Definition 4.12. Assume that $\sigma$ is positive at $P$. If $a \in \operatorname{Sym}(A, \sigma)$, its $F_{P^{-}}$ eigenvalues are defined to be the right eigenvalues (cf. Appendix A) of the matrix $f_{F_{P}}(a \otimes 1) \in M_{n_{P}}\left(D_{P}\right)$. Note that they belong to $F_{P}$ since $f_{F_{P}}(a \otimes 1) \in$ $\operatorname{Sym}\left(M_{n_{P}}\left(D_{P}\right),{ }^{-t}\right)$.

Proposition 4.13. Assume that $1 \in \mathscr{P}$. Let $a \in \operatorname{Sym}(A, \sigma)$. Then $a \in \mathscr{P}$ if and only if all $F_{P}$-eigenvalues of a are nonnegative. Consequently, $1-a \in \mathscr{P}$ if and only if all $F_{P}$-eigenvalues of a are $\leqslant 1$.

Proof. By Proposition 4.6, the positive cone $\mathscr{P}$ extends to a positive cone $\mathscr{P}^{\prime}$ on $\left(A \otimes_{F} F_{P}, \sigma \otimes \mathrm{id}\right)$ over $P^{\prime}$, the unique ordering of $F_{P}$, which is carried by $f_{F_{P}}$ to the positive cone $f_{F_{P}}\left(\mathscr{P}^{\prime}\right)$ on $\left(M_{n_{P}}\left(D_{P}\right),{ }^{-t}\right.$ ) over $P^{\prime}$ (cf. Proposition 4.10 for the notation). By Example 4.3 and since $1 \in \mathscr{P}, f_{F_{P}}\left(\mathscr{P}^{\prime}\right)$ must be $\operatorname{PSD}_{n_{P}}\left(D_{P}, P^{\prime}\right)$. The result follows since $a \in \mathscr{P}$ if and only if $f_{F_{P}}(a \otimes 1) \in \operatorname{PSD}_{n_{P}}\left(D_{P}, P^{\prime}\right)$.

Proposition 4.14. Assume that $F$ is real closed (thus $P$ is its unique ordering) and that $1 \in \mathscr{P}$. Then $\mathscr{P}$ is the set of all hermitian squares of $(A, \sigma)$.

Proof. This follows directly from [4, Cor. 7.15 and Thm. 7.5]. Alternatively, a more explicit proof is as follows: By Remark 4.11(1) and Proposition 4.10, $(A, \sigma) \cong\left(M_{m}(D),{ }^{t}\right)$ where $m \in \mathbb{N}$ and $D \in\left\{F, F(\sqrt{-1}),(-1,-1)_{F}\right\}$. By [4, Thm. 7.5] there is a unique positive cone on $\left(M_{m}(D),{ }^{-t}\right)$ over $P$ containing 1 , and by Example 4.3 this positive cone must be $\operatorname{PSD}_{m}(D, P)$. The result follows since every element of $\operatorname{PSD}_{m}(D, P)$ is a hermitian square (the necessary results for the standard argument in the quaternion case can be found in Appendix A).

4.2. Positive cones on finite-dimensional semisimple algebras with involution. We assume in this section that $A$ is a finite-dimensional semisimple $F$-algebra equipped with an $F$-linear involution $\sigma$. By [11, 1.2.8] we may assume that

$$
(A, \sigma)=\left(A_{1}, \sigma_{1}\right) \times \cdots \times\left(A_{r}, \sigma_{r}\right) \times\left(B_{1}, \tau_{1}\right) \times \cdots \times\left(B_{s}, \tau_{s}\right),
$$

where each $A_{i}$ is a simple $F$-algebra with $F$-linear involution $\sigma_{i}$, and each $B_{j}$ is equal to $C_{j} \times C_{j}^{\text {op }}$ where $C_{j}$ is a simple $F$-algebra and $\tau_{j}(x, y)=(y, x)$ is the $(F$-linear) exchange involution. 
For $1 \leqslant i \leqslant r+s$, we denote by $e_{i}$ the element $(0, \ldots, 0,1,0, \ldots, 0)$ of $A$ with 1 in the $i$-th position.

Proposition 4.15. Let $\mathscr{P}$ be a prepositive cone on $(A, \sigma)$ over $P \in X_{F}$. Then

$$
\mathscr{P}=\mathscr{P}_{1} \times \cdots \times \mathscr{P}_{r} \times \underbrace{\{0\} \times \cdots \times\{0\}}_{s \text { times }},
$$

where each $\mathscr{P}_{i}$ is either a prepositive cone on $\left(A_{i}, \sigma_{i}\right)$ over $P$, or $\{0\}$, and at least one of $\mathscr{P}_{1}, \ldots, \mathscr{P}_{r}$ is not $\{0\}$. Furthermore, if $\mathscr{P}$ is a positive cone, then all $\mathscr{P}_{i}$ are positive cones.

Proof. For $1 \leqslant i \leqslant r+s$ let $\mathscr{P}_{i}=\sigma\left(e_{i}\right) \mathscr{P} e_{i}$ (identifying $\sigma\left(e_{i}\right) \mathscr{P} e_{i}$ with the corresponding subset of $\left.A_{i}\right)$. Obviously $\mathscr{P}_{i} \subseteq \operatorname{Sym}\left(A_{i}, \sigma_{i}\right)$ for $1 \leqslant i \leqslant r$, and it is immediate that $\mathscr{P}_{i}$ satisfies (P1), (P2), (P3), and also (P5) (since $\mathscr{P}_{i} \subseteq \mathscr{P}$ ). Furthermore $P \subseteq\left(\mathscr{P}_{i}\right)_{F}$ and by Remark 4.2 we have either $\mathscr{P}_{i}=\{0\}$ or $\mathscr{P}_{i}$ is a prepositive cone on $\left(A_{i}, \sigma_{i}\right)$ over $P$. Now let $1 \leqslant i \leqslant s$. Then $\mathscr{P}_{r+i}=\{0\}$, since otherwise it would be a prepositive cone on $\left(B_{i}, \tau_{i}\right)$, contradicting [4, Rem. 3.3]. Since, for $a \in \mathscr{P}$, we have $a=\left(\sigma\left(e_{1}\right) a e_{1}, \cdots, \sigma\left(e_{r+s}\right) a e_{r+s}\right)$ (identifying $\sigma\left(e_{i}\right) a e_{i}$ with the corresponding element of $\left.A_{i}\right)$, we obtain $\mathscr{P}=\mathscr{P}_{1} \times \cdots \times \mathscr{P}_{r} \times\{0\} \times \cdots \times$ $\{0\}$. Finally, one of $\mathscr{P}_{1}, \ldots, \mathscr{P}_{r}$ must be different from $\{0\}$, otherwise $\mathscr{P}=\{0\}$, contradicting property $(\mathrm{P} 4)$. The final statement of the proposition is clear.

Corollary 4.16. Let $\mathscr{P}$ be a positive cone on $(A, \sigma)$ with $1 \in \mathscr{P}$. Then $s=0$ and

$$
\mathscr{P}=\mathscr{P}_{1} \times \cdots \times \mathscr{P}_{r}
$$

where each $\mathscr{P}_{i}$ is a positive cone on $\left(A_{i}, \sigma_{i}\right)$ over $P$ with $1 \in \mathscr{P}_{i}$.

Furthermore, if $F$ is real closed then $\mathscr{P}$ is equal to the set of hermitian squares in $(A, \sigma)$.

Proof. The first part of the statement is a direct consequence of Proposition 4.15.

For the second part: since $F$ is real closed and $Z\left(A_{i}\right)$ is a field that is algebraic over $F$ (since $A$ is finite-dimensional over $F$ ), we have $\left[Z\left(A_{i}\right): F\right] \leqslant 2$.

It follows that $F=\operatorname{Sym}\left(A_{i}, \sigma_{i}\right) \cap Z\left(A_{i}\right)$. Indeed, if $Z\left(A_{i}\right)=F$, this is clear. So assume that $Z\left(A_{i}\right)=F(\sqrt{-1})$. If $\sigma_{i}(\sqrt{-1})=\sqrt{-1}$, then $-1 \in \mathscr{P}$, a contradiction. Thus $F(\sqrt{-1})$ is not contained in $\operatorname{Sym}\left(A_{i}, \sigma_{i}\right)$ and so $\operatorname{Sym}\left(A_{i}, \sigma_{i}\right) \cap F(\sqrt{-1})$ is a field of index 2 in $Z\left(A_{i}\right)$ that contains $F$, and so must equal $F$.

Then, by Proposition 4.14, the elements of each $\mathscr{P}_{i}$ are hermitian squares, and the result follows.

Lemma 4.17. Let $\mathscr{P}$ be a positive cone on $(A, \sigma)$. Then:

(1) If $\sum_{i=1}^{\ell} a_{i}=0$ with $a_{1}, \ldots, a_{\ell} \in \mathscr{P}$, then $a_{i}=0$ for all $i$.

(2) If $1 \in \mathscr{P}$, then $\sigma(a) a=0$ implies $a=0$ for all $a \in A$.

Proof. (1) This follows from property (P5).

(2) By Corollary 4.16, $1 \in \mathscr{P}_{i}$ for $1 \leqslant i \leqslant r$ and the result follows from the fact that the forms $\langle 1\rangle_{\sigma_{i}}$ are anisotropic: indeed, if $\langle 1\rangle_{\sigma_{i}}$ were isotropic, then $\ell \times\langle 1\rangle_{\sigma_{i}}$ 
would be universal for some $\ell \in \mathbb{N}$ by [4, Lemma 2.3], and so $D_{\left(A_{i}, \sigma_{i}\right)}\left(\ell \times\langle 1\rangle_{\sigma_{i}}\right)=$ $\operatorname{Sym}\left(A_{i}, \sigma_{i}\right) \subseteq \mathscr{P}_{i}$, contradicting that $\mathscr{P}_{i}$ is proper.

Lemma 4.18. Let $\mathscr{P}$ be a prepositive cone on $(A, \sigma)$ over $P \in X_{F}$ such that $1 \in \mathscr{P}$. Then there is a unique positive cone on $(A, \sigma)$ over $P$ containing 1 , which then contains $\mathscr{P}$.

Proof. Let $\mathscr{Q}$ be a positive cone on $(A, \sigma)$ over $P$ containing 1 . By Proposition 4.6, $\mathscr{Q}=\mathscr{Q}^{\prime} \cap A$, where $\mathscr{Q}^{\prime}$ is a positive cone on $\left(A \otimes_{F} F_{P}, \sigma \otimes \mathrm{id}\right)$, and is thus equal to the set of hermitian squares of $\left(A \otimes_{F} F_{P}, \sigma \otimes \mathrm{id}\right)$ by the final part of Corollary 4.16. Therefore, $\mathscr{Q}$ is the intersection of $A$ with the set of hermitian squares of $\left(A \otimes_{F} F_{P}, \sigma \otimes \mathrm{id}\right)$, so only depends on $A$ and $P$.

Proposition 4.19. Let $\mathscr{P}$ be a positive cone on $(A, \sigma)$ over $P \in X_{F}$ such that $1 \in \mathscr{P}$. Then

$$
\mathscr{P}=\mathscr{C}\left(\mathscr{P}^{\times}\right)
$$

Proof. With the same notation as in Corollary 4.16 we write $\mathscr{P}=\mathscr{P}_{1} \times \cdots \times \mathscr{P}_{r}$ with $\mathscr{P}_{i}$ positive cone on $\left(A_{i}, \sigma_{i}\right)$ over $P$. By Proposition $4.7, \mathscr{P}_{i}$ is a positive cone on $\left(A_{i}, \sigma_{i}\right)$ over some ordering $Q_{i}$ of $Z\left(A_{i}\right) \cap \operatorname{Sym}\left(A_{i}, \sigma_{i}\right)$.

By Lemma 4.9, $\mathscr{P}_{i}=\mathscr{C}\left(\mathscr{P}_{i} \cap A_{i}^{\times}\right)$. Therefore, if $p=\left(p_{1}, \ldots, p_{r}\right) \in \mathscr{P}_{1} \times \cdots \times \mathscr{P}_{r}$ we have, for every $i=1, \ldots, r$,

$$
p_{i}=\sum_{j=1}^{n} \sigma_{i}\left(x_{i j}\right) b_{i j} x_{i j}
$$

for some $n \in \mathbb{N}$ (we can take the same $n$ for all $i$ ), $x_{i j} \in A_{i}$ and $b_{i j} \in \mathscr{P}_{i} \cap A_{i}^{\times}$. Consequently

$$
p=\sum_{j=1}^{n} \sigma\left(\left(x_{1 j}, \ldots, x_{r j}\right)\right)\left(b_{1 j}, \ldots, b_{r j}\right)\left(x_{1 j}, \ldots, x_{r j}\right),
$$

with $\left(b_{1 j}, \ldots, b_{r j}\right) \in \mathscr{P} \cap A^{\times}$, proving the result.

\section{Gauges from Positive CONES}

In this section we construct gauges from positive cones in a natural way, inspired by the classical case of valuations and orderings.

Let $A$ be an $F$-algebra, $\sigma$ an $F$-linear involution on $A, \mathscr{P}$ a prepositive cone on $(A, \sigma)$ over $P \in X_{F}$, and $k$ a subfield of $F$. Following Holland [9, §4], we define the following:

$$
\begin{aligned}
R_{k, \mathscr{P}} & :=\{a \in A \mid \exists m \in k \cap P \sigma(a) a \leqslant \mathscr{P} m\}, \\
I_{k, \mathscr{P}} & :=\left\{a \in A \mid \forall \varepsilon \in k^{\times} \cap P \sigma(a) a \leqslant \mathscr{P} \varepsilon\right\} .
\end{aligned}
$$

Our general strategy is as follows: we first construct a gauge corresponding to $R_{k, \mathscr{P}}$ for some specific algebra with involution, cf. Section 5.1. We then address 
the general case by first reducing to the special case via a scalar extension, and then by showing that the restriction of the gauge thus obtained is still a gauge.

Lemmas 5.1 and 5.2 below are reformulations of Holland's results in our context, using the notation $n(a)$ for $\sigma(a) a$.

Lemma 5.1. Assume that $1 \in \mathscr{P}$ and let $a, b \in A$. Then

(1) $n(a) \geqslant \mathscr{P} 0$;

(2) $n(a+b) \leqslant \mathscr{P} 2(n(a)+n(b))$;

(3) $n(a b)=\sigma(b) n(a) b$;

(4) if a is invertible, then $n\left(a^{-1}\right)=n(\sigma(a))^{-1}$.

Proof. (1) Since $1 \in \mathscr{P}$, we have $\sigma(a) a \in \mathscr{P}$.

(2) It suffices to note that $2(n(a)+n(b))-n(a+b)=n(a-b) \in \mathscr{P}$ as $1 \in \mathscr{P}$.

(3) and (4) are direct.

Lemma 5.2. Assume that $1 \in \mathscr{P}$. Then $R_{k, \mathscr{P}}$ is a subring of $A$ and $I_{k, \mathscr{P}}$ is an ideal of $R_{k, \mathscr{P}}$.

Proof. By definition, $R_{k, \mathscr{P}}$ contains 1 and is closed under multiplication by -1 . It is also clear from Lemma 5.1(2) that $R_{k, \mathscr{P}}$ and $I_{k, \mathscr{P}}$ are closed under sums. Note that for $r, s \in k^{\times} \cap P$ and $a, b \in A$, if (i) $r-n(a) \in \mathscr{P}$ and $s-n(b) \in \mathscr{P}$, then (ii) $r s-n(a b)=r(s-n(b))+\sigma(b)(r-n(a)) b \in \mathscr{P}$. So, if $a, b \in R_{k, \mathscr{P}}$, there exist $r, s$ satisfying (i); then (ii) shows $a b \in R_{k, \mathscr{P}}$. Likewise, if $a \in R_{k, \mathscr{P}}$ and $b \in I_{k, \mathscr{P}}$ then (i) holds for some $r$ and all $s$, so (ii) shows $a b \in I_{k, \mathscr{P}}$.

\section{Remark 5.3.}

(1) The hypothesis $1 \in \mathscr{P}$ in Lemma 5.2 is necessary since, by definition of $I_{k, \mathscr{P}}$, to have $0 \in I_{k, \mathscr{P}}$ we must have $1 \in \mathscr{P}$.

(2) If $1 \in \mathscr{P}$, we have $\mathscr{P} \cap F=P$ (cf. [4, Prop. 3.8(1)], which also holds if $(A, \sigma)$ is an $F$-algebra with $F$-linear involution), and it follows from the definition of $R_{k, \mathscr{P}}$ that $R_{k, \mathscr{P}} \cap F=R_{k, P}$ and $I_{k, \mathscr{P}} \cap F=I_{k, P}$.

Lemma 5.4. Assume that $1 \in \mathscr{P}$. Let $u \in A$ be unitary, i.e., $\sigma(u) u=1$. Then $u \in R_{k, \mathscr{P}}^{\times}$.

Proof. We have $\sigma(u) u=1 \in \mathscr{P}$, so $\sigma(u) u \leqslant \mathscr{P} 1$ and thus $u \in R_{k, \mathscr{P}}$. Since $u^{-1}$ is also unitary, we get $u \in R_{k, \mathscr{P}}^{\times}$.

5.1. Special case with index $\leqslant 2$ and conjugate transposition. Let $E$ be one of $F, C:=F(\sqrt{-1})$ or $H:=(-1,-1)_{F}$ and let $(A, \sigma)=\left(M_{n}(E),{ }^{t}\right)$, where denotes the identity on $F$ or conjugation in the remaining cases. Let $n_{E}(x):=\bar{x} x$ for $x \in E$. Let $P \in X_{F}$ and let $v: F \rightarrow \Gamma_{v} \cup\{\infty\}$ be a valuation on $F$, compatible with $P$.

Lemma 5.5. The function $v_{E}$ defined by

$$
v_{E}(x):=\frac{1}{2} v\left(n_{E}(x)\right)
$$


is a valuation on $E$ and a tame $v$-gauge on $E$. It is the unique extension of $v$ to E.

Proof. Let $F^{h}$ be a Henselization of $F$ with respect to $v$. Since we can take $F^{h} \subseteq F_{P}, H \otimes_{F} F^{h}$ is a division algebra. Therefore, $v$ extends to a valuation on $H$ by [25, Thm. 2.3]. By [25, Thm. 2.1 and (2.7)] this extension is unique and equals $v_{H}$; furthermore, $v$ then extends uniquely to $C$ and this extension equals $v_{C}$. The fact that $v_{E}$ is a gauge follows when $E=C$ from [22, Cor. 1.10], and when $E=H$ from [22, Prop. 1.12], and it is tame by Remark 3.7.

Lemma 5.6. For all $x_{1}, \ldots, x_{r} \in E \backslash\{0\}$ we have

$$
v_{E}\left(n_{E}\left(x_{1}\right)+\cdots+n_{E}\left(x_{r}\right)\right)=2 \min \left\{v_{E}\left(x_{1}\right), \ldots, v_{E}\left(x_{r}\right)\right\} .
$$

Proof. Since each $n_{E}\left(x_{i}\right) \in P$ and $v$ is compatible with $P$, we have

$$
\begin{aligned}
v_{E}\left(n_{E}\left(x_{1}\right)+\cdots+n_{E}\left(x_{r}\right)\right) & =v\left(n_{E}\left(x_{1}\right)+\cdots+n_{E}\left(x_{r}\right)\right) \\
& =\min \left\{v\left(n_{E}\left(x_{1}\right)\right), \ldots, v\left(n_{E}\left(x_{r}\right)\right)\right\} \\
& =2 \min \left\{v_{E}\left(x_{1}\right), \ldots, v_{E}\left(x_{r}\right)\right\} .
\end{aligned}
$$

We define a function $w: M_{n}(E) \rightarrow \Gamma_{v_{E}} \cup\{\infty\}$ as follows, for $a=\left(a_{i j}\right)_{i, j} \in$ $M_{n}(E)$,

$$
w(a):=\min _{i, j}\left\{v_{E}\left(a_{i j}\right)\right\} .
$$

Remark 5.7. By definition of $w, R_{w}=M_{n}\left(R_{v_{E}}\right)$ and $I_{w}=M_{n}\left(I_{v_{E}}\right)$. It follows that $R_{w} / I_{w}=M_{n}\left(R_{v_{E}} / I_{v_{E}}\right)$ is simple.

Lemma 5.8. $w$ is $a^{-t}$-special v-gauge on $M_{n}(E)$. It is the unique ${ }^{-t}$-invariant $v$-gauge on $M_{n}(E)$.

Proof. Straightforward computations show that $w$ is a surmultiplicative $v$-value function. It is also a $v$-norm: let $\left\{e_{i j}\right\}$ be the standard $F$-basis of $M_{n}(F)$ and let $\left\{q_{\ell}\right\}$ be a splitting basis for the $v$-norm $v_{E}$. Then a direct verification shows that $\left\{q_{\ell} e_{i j}\right\}$ is a splitting basis for the $v$-value function $w$.

As observed in Remark 5.7. $\left(M_{n}(E)\right)_{0}=R_{w} / I_{w}$ is simple. Furthermore, since $\Gamma_{w}=\Gamma_{v_{E}}$ and $v_{E}$ is a valuation, it follows that $\Gamma_{w}^{\times}=\Gamma_{w}$. Therefore, by [24, Lemma 2.13], $\operatorname{gr}_{w}\left(M_{n}(E)\right)$ is semisimple, and therefore $w$ is a $v$-gauge.

To show that $w$ is ${ }^{-t}$-special, we use [23, Prop. 1.1]. It is clearly a ${ }^{-t}$-invariant gauge. A direct computation yields $\left(M_{n}(E)_{0},\left({ }^{t}\right)_{0}\right) \cong\left(M_{n}\left(E_{0}\right),(-)_{0}{ }^{t}\right)$, where $\left(E_{0},(-)_{0}\right)$ is one of $\left.\left(F_{0}, \mathrm{id}\right),\left(F_{0}(\sqrt{-1}),-\right),(-1,-1)_{F_{0}},-\right)$. In all three cases the involution $\left({ }^{t}\right)_{0}$ is anisotropic (since hermitian squares in $\left(E_{0},(-)_{0}\right)$ are sums of squares in $F_{0}$ in all three cases and $F_{0}$ is formally real), from which it follows that $\widetilde{(-t)}$ is anisotropic by [23, Cor. 2.3 and Rem. 2.5]. Thus $w$ is ${ }^{-t}$-special by [23, Prop. 1.1].

The uniqueness follows from Proposition 3.8 . 
Lemma 5.9. Let $g$ be $a^{-t}$-special surmultiplicative value function on $M_{n}(E)$, and let $u \in M_{n}(E)$ be unitary, i.e, $\bar{u}^{t} u=1$, then $g(u)=g\left(u^{-1}\right)=0$ and $g\left(u_{a u}^{-1}\right)=$ $g(a)$ for any $a \in M_{n}(E)$.

Proof. If $u$ is unitary in $M_{n}(E)$, then $g\left(\bar{u}^{t} u\right)=0$. Thus, since $g$ is ${ }^{-t}$-special, $g(u)=0$. Similarly, $g\left(u^{-1}\right)=0$. Using surmultiplicativity, it follows that $g\left(u a u^{-1}\right)=g(a)$ for any $a \in M_{n}(E)$.

Let $\mathscr{P}$ be a positive cone on $(A, \sigma)=\left(M_{n}(E),{ }^{-t}\right)$ over $P$ such that $1 \in \mathscr{P}$. Note that $\mathscr{P}$ is then equal to $\operatorname{PSD}_{n}(E, P)$ by Example 4.3 and extends to the positive cone $\operatorname{PSD}_{n}\left(E \otimes_{F} F_{P}, P^{\prime}\right)$ on $\left(A \otimes_{F} F_{P}, \sigma \otimes \mathrm{id}\right) \cong\left(M_{n}\left(E \otimes_{F} F_{P}\right),{ }^{-t}\right)$, where $P^{\prime}$ is the unique ordering on $F_{P}$. Since $v$ is compatible with $P$, there exists a subfield $k$ of $F$ such that $v=v_{k, P}$. Consider the valuation $v^{\prime}=v_{k, P^{\prime}}$ that extends $v$ to $F_{P}$.

The following lemma was suggested to us by A. Wadsworth and simplifies a number of arguments in the paper.

Lemma 5.10. Let $b \in \operatorname{Sym}\left(M_{n}(E),{ }^{-t}\right)$, and let $\lambda_{1}, \ldots, \lambda_{n}$ be the $F_{P}$-eigenvalues of $b$. Then

(1) $w(b)=\min \left\{v^{\prime}\left(\lambda_{1}\right), \ldots, v^{\prime}\left(\lambda_{n}\right)\right\}$;

(2) if $b \in \mathscr{P}$, then $w(b) \leqslant \min \left\{v\left(\bar{x}^{t} b x\right)-v\left(\bar{x}^{t} x\right) \mid x \in E^{n} \backslash\{0\}\right\}$, with equality if $\lambda_{1}, \ldots, \lambda_{n} \in F$

(3) if $b \in \mathscr{P}$, and if $v^{\prime}\left(\lambda_{1}\right)=\cdots=v^{\prime}\left(\lambda_{n}\right)$, then $v\left(\bar{x}^{t} b x\right)=w(b)+v\left(\bar{x}^{t} x\right)$ for each $x \in E^{n} \backslash\{0\}$.

Proof. For $a=\left(a_{i j}\right)_{i, j} \in M_{n}\left(E \otimes_{F} F_{P}\right)$, let $w^{\prime}(a):=\min _{i, j}\left\{v_{E}^{\prime}\left(a_{i j}\right)\right\}$, where $v_{E}^{\prime}(x)=\frac{1}{2} v^{\prime}\left(n_{E \otimes F_{P}}(x)\right)$ is the unique extension of $v^{\prime}$ to $E \otimes_{F} F_{P}$. Note that $\left.w^{\prime}\right|_{M_{n}(E)}=w$, and that $w^{\prime}$ is a $(-\otimes \mathrm{id})^{t}$-special $v^{\prime}$-gauge on $M_{n}\left(E \otimes_{F} F_{P}\right)$ by Lemma 5.8. It is also immediate that $w^{\prime}$ is a $v_{E}$-value function.

Note that since $b$ is symmetric, $b \otimes 1 \in M_{n}\left(E \otimes_{F} F_{P}\right)$ is diagonalizable by congruences with eigenvalues in $F_{P}$ by the Principal Axis Theorem, cf. Appendix A for the quaternionic case. Therefore, let $u \in M_{n}\left(E \otimes_{F} F_{P}\right)$ be unitary such that $\bar{u}^{t}(b \otimes 1) u=\operatorname{diag}\left(\lambda_{1}, \ldots, \lambda_{n}\right)$ with $\lambda_{1}, \ldots, \lambda_{n} \in F_{P}$.

We now prove the statements.

(1) By Lemma 5.9,

$$
w(b)=w^{\prime}(b \otimes 1)=w^{\prime}\left(\operatorname{diag}\left(\lambda_{1}, \ldots, \lambda_{n}\right)\right)=\min \left\{v^{\prime}\left(\lambda_{1}\right), \ldots, v^{\prime}\left(\lambda_{n}\right)\right\} .
$$

(2) We first show that $w^{\prime}(b \otimes 1) \leqslant v^{\prime}\left(\bar{x}^{t}(b \otimes 1) x\right)-v^{\prime}\left(\bar{x}^{t} x\right)$ for every $x \in$ $\left(E \otimes_{F} F_{P}\right)^{n} \backslash\{0\}$. As observed above, we may assume that $b \otimes 1=\operatorname{diag}\left(\lambda_{1}, \ldots, \lambda_{n}\right)$ with $\lambda_{1}, \ldots, \lambda_{n} \in F_{P}$. Note that since $b \otimes 1 \in \operatorname{PSD}_{n}\left(E \otimes_{F} F_{P}, P^{\prime}\right), \lambda_{1}, \ldots, \lambda_{n} \in P^{\prime}$ and thus, since $v^{\prime}$ is compatible with $P^{\prime}$, we have $v^{\prime}\left(\bar{x}^{t}(b \otimes 1) x\right)=\min _{i}\left\{v^{\prime}\left(\lambda_{i}\right)+\right.$ $\left.v^{\prime}\left(\bar{x}_{i} x_{i}\right)\right\}$ and $v^{\prime}\left(\bar{x}^{t} x\right)=\min _{i}\left\{v^{\prime}\left(\bar{x}_{i} x_{i}\right)\right\}$. With this observation, the inequality holds if $\min _{i}\left\{v^{\prime}\left(\bar{x}_{i} x_{i}\right)\right\}=0$ : it is then equivalent to $\min _{i}\left\{v^{\prime}\left(\lambda_{i}\right)\right\} \leqslant \min _{i}\left\{v^{\prime}\left(\lambda_{i}\right)+\right.$ $\left.v^{\prime}\left(\bar{x}_{i} x_{i}\right)\right\}$, which is true since $v^{\prime}\left(\bar{x}_{i} x_{i}\right) \geqslant 0$ for all $i$. For the general case, let $i_{0}$ be 
such that $\min _{i}\left\{v^{\prime}\left(\bar{x}_{i} x_{i}\right)\right\}=v^{\prime}\left(\bar{x}_{i_{0}} x_{i_{0}}\right)$. Then the inequality is equivalent to

$$
\left.w^{\prime}\left(\overline{x_{i_{0}}^{-1}}(b \otimes 1) x_{i_{0}}^{-1}\right) \leqslant v^{\prime}\left({\overline{\left(x x_{i_{0}}^{-1}\right)}}^{t}(b \otimes 1)\left(x x_{i_{0}}^{-1}\right)\right)-v^{\prime}{\overline{\left(x x_{i_{0}}^{-1}\right)}}^{t}\left(x x_{i_{0}}^{-1}\right)\right),
$$

and we are reduced to the previous case. It then follows that $w(b) \leqslant \min _{i}\left\{v\left(\bar{x}^{t} b x\right)-\right.$ $\left.v\left(\bar{x}^{t} x\right) \mid x \in E^{n} \backslash\{0\}\right\}$.

Assume now that $\lambda_{1}, \ldots, \lambda_{n} \in F$ and let $y_{1}, \ldots, y_{n} \in E^{n} \backslash\{0\}$ be eigenvectors corresponding to these right eigenvalues. Then $v\left(\bar{y}_{i}{ }^{t} b y_{i}\right)-v\left(\bar{y}_{i}{ }^{t} y_{i}\right)=v\left(\lambda_{i}\right)$ and the result follows.

(3) This is a direct computation, using that $v^{\prime}$ is comptatible with $P^{\prime}$ and that $\lambda_{1}, \ldots, \lambda_{n} \in P^{\prime}$ since $b \in \mathscr{P}$. If $\gamma=v^{\prime}\left(\lambda_{1}\right)=\cdots=v^{\prime}\left(\lambda_{n}\right)$, then

$$
\begin{aligned}
v\left(\bar{x}^{t} b x\right) & =v\left(\overline{\bar{u}}^{t} x\right. \\
& =v\left(\bar{u}^{t} \operatorname{diag}\left(\lambda_{1}, \ldots, \lambda_{n}\right) y\right), \quad \text { where } y=\bar{u}^{t} x \\
& =v\left(\sum_{i} \bar{y}_{i} \lambda_{i} y_{i}\right) \\
& =v\left(\sum_{i} \lambda_{i} \bar{y}_{i} y_{i}\right), \quad \text { where the summands are all in } P^{\prime} \\
& =\gamma+\min \left\{v^{\prime}\left(\bar{y}_{i} y_{i}\right)\right\} \\
& =\gamma+v^{\prime}\left(\bar{y}^{t} y\right) \\
& =\gamma+v\left(\bar{x}^{t} x\right) .
\end{aligned}
$$

Proposition 5.11. We have

$$
R_{w}=\left\{a \in M_{n}(E) \mid \text { the } F_{P} \text {-eigenvalues of } \bar{a}^{t} \text { a belong to } R_{v^{\prime}}\right\}=R_{k, \mathscr{P}}
$$

and

$$
I_{w}=\left\{a \in M_{n}(E) \mid \text { the } F_{P} \text {-eigenvalues of } \bar{a}^{t} a \text { belong to } I_{v^{\prime}}\right\}=I_{k, \mathscr{P}} .
$$

Proof. We only check the first statement, the second one is similar. Let $a \in$ $M_{n}(E)$. Then $w(a) \geqslant 0$ if and only if $w\left(\bar{a}^{t} a\right) \geqslant 0$ by Lemma 5.8 and, by Lemma 5.10(1), this is equivalent to all the $F_{P^{-}}$eigenvalues of $\bar{a}^{t} a$ belonging to $R_{v^{\prime}}$, proving the first equality.

Observe now that if $b \in \mathscr{P}$ and $r \in F$ then, using extension of scalars to $F_{P}$ and the fact that $\lambda$ is an $F_{P^{-}}$-eigenvalue of $b$ if and only if $r-\lambda$ is an $F_{P}$-eigenvalue of $r-b$ :

$$
\begin{aligned}
b \leqslant \mathscr{P} r & \Leftrightarrow(r-b) \otimes 1 \in \operatorname{PSD}_{n}\left(E \otimes_{F} F_{P}, P^{\prime}\right) \\
& \Leftrightarrow \text { all } F_{P^{-}} \text {eigenvalues of } r-b \text { are in } P^{\prime} \\
& \Leftrightarrow \lambda \leqslant_{P^{\prime}} r \text { for every } F_{P^{-}} \text {eigenvalue } \lambda \text { of } b .
\end{aligned}
$$

Applying this observation to $\bar{a}^{t} a$ we obtain, for $a \in M_{n}(E)$ : there exists $r \in k \cap P$ such that $\bar{a}^{t} a \leqslant \mathscr{P} r$ if and only if all the $F_{P^{-e i g e n v a l u e s ~ o f ~}} \bar{a}^{t} a$ belong to $R_{v^{\prime}}$, proving the second equality. 
Proposition 5.12. $w$ is the unique v-gauge on $\left(M_{n}(E),{ }^{-t}\right)$ with gauge ring $R_{k, \mathscr{P}}$. Proof. Let $g$ be a $v$-gauge on $\left(M_{n}(E),{ }^{-t}\right)$ with gauge $\operatorname{ring} R_{k, \mathscr{P}}$. Then $I_{g}=$ $J\left(R_{k, \mathscr{P}}\right)=I_{w}$ by Remark 3.4 and thus $R_{g} / I_{g}$ is simple, cf. Remark 5.7. In this case, by [24, Prop. 3.48], $g$ is completely determined by $R_{g}=R_{w}$.

Definition 5.13. Following Proposition 5.12 , we define $w_{k, \mathscr{P}}:=w$ on $\left(M_{n}(E),{ }^{t}\right)$.

5.2. The general case. Assume now that $A$ is a finite-dimensional simple $F$ algebra with $F$-linear involution $\sigma$ such that $F=\operatorname{Sym}(A, \sigma) \cap Z(A)$, and that $\mathscr{P}$ is a positive cone on $(A, \sigma)$ over $P \in X_{F}$ such that $1 \in \mathscr{P}$.

\section{Remark 5.14.}

(1) Recall from [4, Cor. 7.7] that $1 \in \mathscr{P}$ means that the involution $\sigma$ is positive at $P$.

(2) If the hypothesis $1 \in \mathscr{P}$ is not satisfied, it is possible to regain it, at the cost of changing the involution (and the positive cone): Take $a \in \mathscr{P}^{\times}$(it exists by [4, Lemma 3.6]). Then $a^{-1} \mathscr{P}$ is a positive cone on $\left(A\right.$, $\left.\operatorname{Int}\left(a^{-1}\right) \circ \sigma\right)$ over $P$.

Since $1 \in \mathscr{P}, \sigma$ is positive at $P$ by Remark $4.11(1)$. Let $f_{F_{P}}:\left(A \otimes_{F} F_{P}, \sigma \otimes\right.$ id $\stackrel{\sim}{\longrightarrow}\left(M_{n_{P}}\left(D_{P}\right),{ }^{-t}\right)$, with $n_{P}$ and $D_{P}$ as in Proposition 4.10 and $f_{F_{P}}$ as in Remark 4.11(2). Let $P^{\prime}$ be the unique ordering on $F_{P}$ and let $\mathscr{P}^{\prime}$ be the unique positive cone on $\left(A \otimes_{F} F_{P}, \sigma \otimes\right.$ id) over $P^{\prime}$ that extends $\mathscr{P}$, cf. Proposition 4.6, i.e., $\mathscr{P}^{\prime} \cap A=\mathscr{P}$. Let $\mathscr{Q}=f_{F_{P}}\left(\mathscr{P}^{\prime}\right)$ and note that $1 \in \mathscr{Q}$. As observed in the proof of Proposition 4.13, $\mathscr{Q}=\operatorname{PSD}_{n_{P}}\left(D_{P}, P^{\prime}\right)$.

Consider the diagram

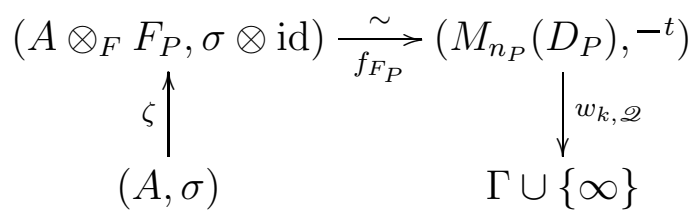

where $\zeta$ is the canonical morphism $a \mapsto a \otimes 1$, and $w_{k, \mathscr{Q}}$ is the gauge defined on $M_{n_{P}}\left(D_{P}\right)$ obtained from $\mathscr{Q}$, cf. Definition 5.13. We define

$$
w_{k, \mathscr{P}}:=w_{k, \mathscr{Q}} \circ f_{F_{P}} \circ \zeta .
$$

Thus, $w_{k, \mathscr{P}}(a)=w_{k, \mathscr{Q}}\left(f_{F_{P}}(a \otimes 1)\right)$.

A priori $w_{k, \mathscr{P}}$ depends on $f_{F_{P}}$. However, we will show in Theorem 5.16 that it is a $\sigma$-special $v_{k, P}$-gauge and therefore unique.

Proposition 5.15. $R_{w_{k, \mathscr{P}}}=R_{k, \mathscr{P}}$ and $I_{w_{k, \mathscr{P}}}=I_{k, \mathscr{P}}$.

Proof. Since $\mathscr{P}^{\prime}$ is an extension of $\mathscr{P}$, we have $R_{k, \mathscr{P}} \cap A=R_{k, \mathscr{P}}$ and, since $f_{F_{P}}\left(\mathscr{P}^{\prime}\right)=\mathscr{Q}$, we have $f_{F_{P}}\left(R_{k, \mathscr{P}^{\prime}}\right)=R_{k, \mathscr{Q}}$. Therefore, for $a \in A$ :

$$
\begin{aligned}
a \in R_{k, \mathscr{P}} & \Leftrightarrow a \otimes 1 \in R_{k, \mathscr{P} \prime} \\
& \Leftrightarrow f_{F_{P}}(a \otimes 1) \in R_{k, \mathscr{Q}}
\end{aligned}
$$




$$
\begin{aligned}
& \Leftrightarrow w_{k, \mathscr{Q}}\left(f_{F_{P}}(a \otimes 1)\right) \geqslant 0 \quad \text { [by Proposition 5.11] } \\
& \Leftrightarrow w_{k, \mathscr{P}}(a) \geqslant 0,
\end{aligned}
$$

and similarly for $I_{k, \mathscr{P}}$.

Theorem 5.16. The map $w_{k, \mathscr{P}}$ is a $\sigma$-special $v_{k, P}$-gauge on $A$. It is tame, and it is the only $\sigma$-invariant $v_{k, P}$-gauge on $A$.

Proof. Let $v^{\prime}$ be the valuation $v_{k, P^{\prime}}$ on $F_{P}$ and note that it extends $v_{k, P}$.

By Lemma 4.17(2), $\sigma \otimes \mathrm{id}_{F_{P}}$ is anisotropic since $\mathscr{P}^{\prime}$ is a positive cone containing 1. Let $F^{h}$ denote a Henselization of $F$ with respect to $v_{k, P}$. Since $\left(F_{P}, v^{\prime}\right)$ is Henselian, we may assume that $F^{h} \subseteq F_{P}$ and obtain in particular that $\sigma \otimes \mathrm{id}_{F^{h}}$ is anisotropic. It then follows from Proposition 3.8(1) and Remark 3.7 that there is a $\sigma$-special tame $v_{k, P}$-gauge $g$ on $A$. Therefore, by [22, Cor. 1.26] and Remark 3.7, $g \otimes v^{\prime}$ is a (tame) $v^{\prime}$-gauge on $A \otimes_{F} F_{P}$, and by [23, Cor. 1.4] it is $\sigma \otimes \operatorname{id}_{F_{P}}$-invariant. Since $w_{k, \mathscr{Q}}$ is a ${ }^{-t}$-special $v^{\prime}$-gauge (cf. Lemma [5.8), $w_{k, \mathscr{Q}} \circ f_{F_{P}}$ is a $\sigma \otimes$ id-special $v^{\prime}$-gauge on $A \otimes_{F} F_{P}$. Therefore, by Proposition 3.8 (2), $w_{k, \mathscr{Q}} \circ f_{F_{P}}=g \otimes v^{\prime}$ and it follows that $w_{k, \mathscr{Q}} \circ f_{F_{P}} \circ \zeta=g$, i.e., $g=w_{k, \mathscr{P}}$. Thus, $w_{k, \mathscr{P}}$ is a $\sigma$-special (tame) $v_{k, P^{-g a u g e . ~}}$

The uniqueness statement follows from Proposition 3.8(2).

We finish this section with a description of the elements in

$$
\operatorname{st}\left(w_{k, \mathscr{P}}\right):=\left\{a \in A^{\times} \mid w_{k, \mathscr{P}}\left(a^{-1}\right)=-w_{k, \mathscr{P}}(a)\right\},
$$

cf. [22, p. 709] (and also [22, Lemma 1.3] for some properties of the set $\operatorname{st}\left(w_{k, \mathscr{P}}\right)$ ):

\section{Proposition 5.17.}

(1) Let $a \in \operatorname{Sym}(A, \sigma)^{\times}$. Then $a \in \operatorname{st}\left(w_{k, \mathscr{P}}\right)$ if and only if all $F_{P^{-}}$-eigenvalues of a have the same $v_{k, P}$-value.

(2) Let $a \in A^{\times}$. Then $a \in \operatorname{st}\left(w_{k, \mathscr{P}}\right)$ if and only if all $F_{P^{-} \text {-eigenvalues of } \sigma(a) \text { a have }}$ the same $v_{k, P}$-value.

Proof. We denote the unique ordering on $F_{P}$ by $P^{\prime}, v_{k, P^{\prime}}$ by $v^{\prime}$ and $w_{k, \mathscr{P}}$ by $w$.

(1) Let $\lambda_{1}, \ldots, \lambda_{n}$ be the $F_{P}$-eigenvalues of $a$, ordered such that $v^{\prime}\left(\lambda_{1}\right) \leqslant \cdots \leqslant$ $v^{\prime}\left(\lambda_{n}\right)$ (so that $w(a)=v^{\prime}\left(\lambda_{1}\right)$ by Lemma 5.10(1)).

The $F_{P^{-}}$eigenvalues of $a^{-1}$ are $\lambda_{1}^{-1}, \ldots, \lambda_{n}^{-1}$, and have values $-v^{\prime}\left(\lambda_{n}\right) \leqslant \cdots \leqslant$ $-v^{\prime}\left(\lambda_{1}\right)$, so that $w\left(a^{-1}\right)=-v^{\prime}\left(\lambda_{n}\right)$. Therefore $w(a)=-w\left(a^{-1}\right)$ if and only if $v^{\prime}\left(\lambda_{1}\right)=v^{\prime}\left(\lambda_{n}\right)$ if and only if $v^{\prime}\left(\lambda_{1}\right)=\cdots=v^{\prime}\left(\lambda_{n}\right)$.

(2) Since $w$ is $\sigma$-special, we have the equivalences

$$
\begin{aligned}
w\left(a^{-1}\right)=-w(a) & \Leftrightarrow 2 w\left(a^{-1}\right)=-2 w(a) \Leftrightarrow w\left(a^{-1} \sigma\left(a^{-1}\right)\right)=-w(\sigma(a) a) \\
& \Leftrightarrow w\left((\sigma(a) a)^{-1}\right)=-w(\sigma(a) a)
\end{aligned}
$$

and the result follows from (1) since $\sigma(a) a$ is symmetric. 
5.3. Explicit computation in the special case with index $\leqslant 2$ and arbitrary involution. The content of this section is partially inspired by a talk of J.-P. Tignol at the 2009 conference "Positivity, Valuations, and Quadratic Forms" at the University of Konstanz.

Let $(A, \sigma)=\left(M_{n}(E), \operatorname{ad}_{h}\right)$ with $(E,-)$ as in Section 5.1 and $h=\left\langle e_{1}, e_{2}, \ldots, e_{n}\right\rangle_{-}$, where $e_{1}, \ldots, e_{n} \in F^{\times}=\operatorname{Sym}(E,-) \backslash\{0\}$. Let $P \in X_{F}$ and let $\mathscr{P}$ be a positive cone on $(A, \sigma)$ over $P$ and with $1 \in \mathscr{P}$. Let $v$ be a valuation on $F$, compatible with $P$ (i.e., $v=v_{k, P}$ for some subfield $k$ of $F$ ) and let $v_{E}$ be the unique extension of $v$ to $E$, cf. Lemma 5.5 .

Let $e=\operatorname{diag}\left(e_{1}, \ldots, e_{n}\right) \in M_{n}(F) \subseteq M_{n}(E)$. Then $\sigma=\operatorname{Int}\left(e^{-1}\right) \circ{ }^{-t}$.

Lemma 5.18. The elements $e_{1}, \ldots, e_{n}$ are all in $P$, or are all in $-P$.

Proof. Since $1 \in \mathscr{P}$, the involution $\operatorname{ad}_{h}$ is positive at $P$ by [4, Cor. 7.7] and so has maximal signature at $P$ by [3, Rem. 4.3]. Since $\operatorname{ad}_{h}=\left({ }^{-t}\right)_{e}$ in the notation of [3, p. 352], the hermitian form $\langle e\rangle_{-}$has maximal signature at $P$ in absolute value by [3. Prop. 4.4(iv)], i.e., the matrix $e$ is positive definite or negative definite at $P$. The statement follows.

Since $\operatorname{ad}_{h}=\operatorname{ad}_{-h}$, we may assume that the $e_{i}$ are all in $P$. It follows that the matrix $e \otimes 1$ has a square root $\sqrt{e \otimes 1}$ in $M_{n}\left(E \otimes_{F} F_{P}\right)$.

Let $\mathscr{P}^{\prime}$ be the unique extension of $\mathscr{P}$ to $\left(A \otimes_{F} F_{P}, \sigma \otimes\right.$ id) over $P^{\prime}$, the unique ordering of $F_{P}$, cf. Proposition 4.6. Consider the valuation $v^{\prime}=v_{k, P^{\prime}}$ that extends $v$ to $F_{P}$ and let $v_{E}^{\prime}$ be the unique extension of $v^{\prime}$ to $E \otimes_{F} F_{P}$, cf. Lemma 5.5.

After canonically identifying $\left(A \otimes_{F} F_{P}, \sigma \otimes\right.$ id $)$ with $\left(M_{n}\left(E \otimes_{F} F_{P}\right), a_{h \otimes 1}\right)$ and noting that $\operatorname{ad}_{h \otimes 1}=\operatorname{Int}\left(e^{-1} \otimes 1\right) \circ^{-t}$, we consider the isomorphism

$$
f=\operatorname{Int}(\sqrt{e \otimes 1}):\left(M_{n}\left(E \otimes_{F} F_{P}\right), \operatorname{Int}\left(e^{-1} \otimes 1\right) \circ^{-t}\right) \stackrel{\sim}{\longrightarrow}\left(M_{n}\left(E \otimes_{F} F_{P}\right),{ }^{-t}\right) .
$$

Then $f\left(\mathscr{P}^{\prime}\right)$ is a positive cone over $P^{\prime}$ containing 1 , and thus $f\left(\mathscr{P}^{\prime}\right)=\operatorname{PSD}_{n}\left(E \otimes_{F}\right.$ $\left.F_{P}, P^{\prime}\right)$, the positive cone of all positive semidefinite $n \times n$-matrices over $E \otimes_{F} F_{P}$, cf. Example 4.3. Following Section 5.2 , the gauge $w_{k, \mathscr{P}}$ is defined via the following diagram:

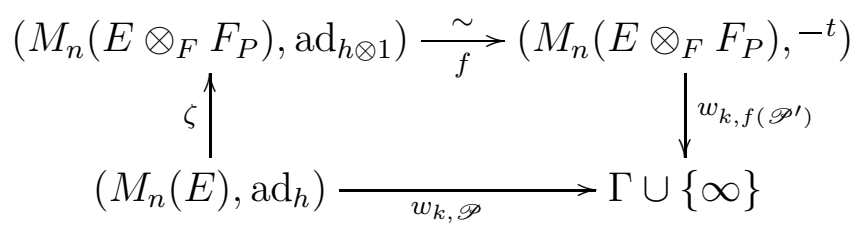

where

$$
w_{k, f\left(\mathscr{P}^{\prime}\right)}\left(\left(x_{i j}\right)_{i, j}\right)=\min _{i, j}\left\{v_{E}^{\prime}\left(x_{i j}\right)\right\}
$$


For $a=\left(a_{i j}\right)_{i, j} \in M_{n}(E)$ we have

$$
\begin{aligned}
w_{k, \mathscr{P}}(a) & =w_{k, f\left(\mathscr{P}^{\prime}\right)}(f(a \otimes 1)) \\
& =\min _{i, j}\left\{v_{E}^{\prime}\left(\sqrt{e_{i} \otimes 1}\left(a_{i j} \otimes 1\right){\sqrt{e_{j} \otimes 1}}^{-1}\right)\right\} \\
& =\min _{i, j}\left\{v_{E}\left(a_{i j}\right)+\frac{1}{2} v\left(e_{i}\right)-\frac{1}{2} v\left(e_{j}\right)\right\} .
\end{aligned}
$$

Therefore

$$
\begin{aligned}
R_{k, \mathscr{P}} & =\left\{\left(a_{i j}\right)_{i, j} \in M_{n}(E) \mid \forall i, j \quad v_{E}\left(a_{i j}\right) \geqslant-\frac{1}{2} v\left(e_{i} e_{j}^{-1}\right)\right\} \\
& =:\left(\left\{v_{E}(x) \geqslant-\frac{1}{2} v\left(e_{i} e_{j}^{-1}\right)\right\}\right)_{i, j} .
\end{aligned}
$$

Similarly, we obtain

$$
\begin{aligned}
I_{k, \mathscr{P}} & =\left\{\left(a_{i j}\right)_{i, j} \in M_{n}(E) \mid \forall i, j \quad v_{E}\left(a_{i j}\right)>-\frac{1}{2} v\left(e_{i} e_{j}^{-1}\right)\right\} \\
& =:\left(\left\{v_{E}(x)>-\frac{1}{2} v\left(e_{i} e_{j}^{-1}\right)\right\}\right)_{i, j} .
\end{aligned}
$$

We conclude that

$$
R_{k, \mathscr{P}} / I_{k, \mathscr{P}}=\left(\left\{v_{E}(x) \geqslant-\frac{1}{2} v\left(e_{i} e_{j}^{-1}\right)\right\} /\left\{v_{E}(x)>-\frac{1}{2} v\left(e_{i} e_{j}^{-1}\right)\right\}\right)_{i, j} .
$$

Observe that if $\frac{1}{2} v\left(e_{i} e_{j}^{-1}\right) \notin \Gamma_{v_{E}}$, i.e., if $v\left(e_{i}\right) \neq v\left(e_{j}\right) \bmod 2 \Gamma_{v_{E}}$, then

$$
\left\{v_{E}(x) \geqslant-\frac{1}{2} v\left(e_{i} e_{j}^{-1}\right)\right\}=\left\{v_{E}(x)>-\frac{1}{2} v\left(e_{i} e_{j}^{-1}\right)\right\}
$$

and so

$$
\left\{v_{E}(x) \geqslant-\frac{1}{2} v\left(e_{i} e_{j}^{-1}\right)\right\} /\left\{v_{E}(x)>-\frac{1}{2} v\left(e_{i} e_{j}^{-1}\right)\right\}=\{0\} .
$$

Larmour [15, §3] defined residue forms of hermitian forms over valued division algebras with involution. We recall what we need from [15]. There are nonzero elements $\pi_{1}, \ldots, \pi_{r} \in \operatorname{Sym}(E,-)=F$, such that $v_{E}\left(\pi_{1}\right)=v\left(\pi_{1}\right), \ldots, v_{E}\left(\pi_{r}\right)=$ $v\left(\pi_{r}\right)$ are all distinct modulo $2 \Gamma_{v_{E}}$, and

$$
h \simeq h_{1} \perp \cdots \perp h_{r}
$$

where

$$
h_{i}=\left\langle u_{i, 1} \pi_{i}, \ldots, u_{i, n_{i}} \pi_{i}\right\rangle_{-}
$$

for units $u_{i, \ell}$ in $R_{v_{E}}$. The residue forms of $h$ are then the forms

$$
\widetilde{h}_{i}=\left\langle u_{i, 1}+I_{v_{E}}, \ldots, u_{i, n_{i}}+I_{v_{E}}\right\rangle_{\vartheta_{i}}
$$

over $\left(R_{v_{E}} / I_{v_{E}}, \vartheta_{i}\right)$, where $\vartheta_{i}$ is the involution induced by $\operatorname{Int}\left(\pi_{i}\right) \circ-=-$ (since $\left.\pi_{i} \in F\right)$.

Proposition 5.19. We have

$$
\left(A_{0}, \sigma_{0}\right) \cong\left(M_{n_{1}}\left(E_{0}\right), \operatorname{ad}_{\widetilde{h}_{1}}\right) \times \cdots \times\left(M_{n_{r}}\left(E_{0}\right), \operatorname{ad}_{\widetilde{h}_{r}}\right)
$$


and

$$
\Gamma_{w_{k, \mathscr{P}}}=\sum_{i, j=1}^{r} \frac{1}{2}\left(v\left(\pi_{i}\right)-v\left(\pi_{j}\right)\right)+\Gamma_{v_{E}} .
$$

Proof. The computation of $\Gamma_{w_{k, \mathscr{P}}}$ is immediate from (5.5). Going back to the notation $h=\left\langle e_{1}, e_{2}, \ldots, e_{n}\right\rangle_{-}$and, writing

$$
h_{1}=\left\langle e_{1}, \ldots, e_{n_{1}}\right\rangle_{-}, h_{2}=\left\langle e_{n_{1}+1}, \ldots, e_{n_{1}+n_{2}}\right\rangle_{-}, \ldots
$$

and

$$
I_{1}=\left\{1, \ldots, n_{1}\right\}, I_{2}=\left\{n_{1}+1, \ldots, n_{1}+n_{2}\right\}, \ldots
$$

we have $v_{E}\left(e_{i}\right)=v_{E}\left(\pi_{\ell}\right)$ for every $i \in I_{\ell}$. Then, using (5.7) and the computation leading to it, we obtain

$$
A_{0}=R_{k, \mathscr{P}} / I_{k, \mathscr{P}} \cong\left(\begin{array}{cccc}
M_{1} & 0 & & \\
0 & M_{2} & & \\
& & \ddots & 0 \\
& & 0 & M_{r}
\end{array}\right) \cong M_{1} \times \cdots \times M_{r}
$$

where, for $\ell=1, \ldots, r$,

$$
\begin{aligned}
M_{\ell} & =\left(\left\{v_{E}(x) \geqslant-\frac{1}{2} v\left(e_{i} e_{j}^{-1}\right)\right\} /\left\{v_{E}(x)>-\frac{1}{2} v\left(e_{i} e_{j}^{-1}\right)\right\}\right)_{i, j \in I_{\ell}} \\
& =\left(\left\{v_{E}(x) \geqslant 0\right\} /\left\{v_{E}(x)>0\right\}\right)_{i, j \in I_{\ell}} \\
& =M_{n_{\ell}}\left(R_{v_{E}} / I_{v_{E}}\right) \\
& =M_{n_{\ell}}\left(E_{0}\right) .
\end{aligned}
$$

Finally, a direct computation shows that $\left.\sigma_{0}\right|_{M_{\ell}}=\operatorname{ad}_{\widetilde{h}_{\ell}}$ for $\ell=1, \ldots, r$.

Corollary 5.20. $h$ has only one residue form if and only if $R_{k, \mathscr{P}}$ is a Dubrovin valuation ring of $M_{n}(E)$.

Proof. Assume that $h$ has only one residue form. Then the proof of Proposition 5.19 together with (5.6) shows that $R_{k, \mathscr{P}}=M_{n}\left(R_{v_{E}}\right)$, which is a Dubrovin valuation ring of $M_{n}(E)$ since $R_{v_{E}}$ is a valuation ring of $E$, cf. [18, p. 606-607].

Conversely, if $R_{k, \mathscr{P}}$ is a Dubrovin valuation ring of $M_{n}(E)$ then, by definition, $R_{k, \mathscr{P}} / I_{k, \mathscr{P}}$ is simple and so $h$ has only one residue form by Proposition 5.19,

\section{Compatibility of Positive CONES AND $\sigma$-invariant Gauges}

In this section we let $A$ be a finite-dimensional simple $F$-algebra with $F$-linear involution $\sigma$ such that $F=\operatorname{Sym}(A, \sigma) \cap Z(A)$, we fix $P \in X_{F}$ and a positive cone $\mathscr{P}$ on $(A, \sigma)$ over $P$ such that $1 \in \mathscr{P}$. Recall that this implies $\mathscr{P} \cap F=P$, cf. Remark 5.3). If $S \subseteq T$ are subsets of $A$ we say that $S$ is $\mathscr{P}$-convex in $T$ if for all $s_{1}, s_{2} \in S$ and $t \in T, s_{1} \leqslant \mathscr{P} t \leqslant \mathscr{P} s_{2}$ implies $t \in S$.

Let $w$ be a $\sigma$-invariant $v$-gauge on $A$. As before, we denote the induced involution on $A_{0}=R_{w} / I_{w}$ by $\sigma_{0}$. Recall that $R_{w} \cap F=R_{v}$ and $I_{w} \cap F=I_{v}$ since 
$\left.w\right|_{F}=v$ and thus that $A_{0}$ is an $F_{v}$-algebra. Denote the canonical projections $R_{v} \rightarrow F_{v}$ and $R_{w} \rightarrow A_{0}$ by $\pi_{v}$ and $\pi_{w}$, respectively. Note that $\pi_{w}$ extends $\pi_{v}$.

Inspired by the classical compatibility conditions between valuations and orderings, we consider the following properties:

(C0) for all $a, b \in \mathscr{P}, w(a+b)=\min \{w(a), w(b)\}$;

(C1) for all $a, b \in A, 0 \leqslant \mathscr{P} a \leqslant \mathscr{P} b$ implies $w(b) \leqslant w(a)$;

(C2) $R_{w}$ is $\mathscr{P}$-convex in $A$;

(C3) $I_{w}$ is $\mathscr{P}$-convex in $A$;

(C4) $I_{w}$ is $\mathscr{P}$-convex in $R_{w}$;

(C5) $\pi_{w}\left(\mathscr{P} \cap R_{w}\right)$ is a prepositive cone on $\left(A_{0}, \sigma_{0}\right)$;

(C6) $a \in \mathscr{P} \cap I_{w} \Rightarrow a<\mathscr{P} 1$;

(C7) $1+\operatorname{Sym}\left(I_{w}, \sigma\right) \subseteq \mathscr{P}$.

It is clear that $(\mathrm{C} 0)$ and $(\mathrm{C} 1)$ are equivalent, that $(\mathrm{C} 1)$ implies $(\mathrm{C} 2)$ and $(\mathrm{C} 3)$, and also that (C3) implies (C4). Using the fact that $w_{k, \mathscr{P}}$ is the unique $\sigma$-invariant $v_{k, P}$-gauge on $A$ (Theorem 5.16), we will show in Propositions 6.5 and 6.7 below that properties $(\mathrm{C} 0), \ldots,(\mathrm{C} 7)$ are equivalent. However, first we would like to point out that the equivalences $(\mathrm{C} 4) \Leftrightarrow(\mathrm{C} 5)$ and $(\mathrm{C} 6) \Leftrightarrow(\mathrm{C} 7)$ can be obtained in an elementary way, using only the definitions of $(\mathrm{C} 0), \ldots,(\mathrm{C} 7)$ :

Proposition 6.1. (C4) implies (C5). More precisely under the hypothesis (C4) we have:

(1) $I_{v}=I_{w} \cap F$ is $P$-convex in $R_{v}=R_{w} \cap F$, and so $\bar{P}:=\pi_{v}\left(P \cap R_{v}\right)$ is an ordering on $F_{v}$.

(2) The set $\pi_{w}\left(\mathscr{P} \cap R_{w}\right)$ is a prepositive cone on $\left(A_{0}, \sigma_{0}\right)$ over $\bar{P}$.

Proof. (1) Let $a \in R_{w} \cap F$ and $b \in I_{w} \cap F$ be such that $0 \leqslant_{P} a \leqslant_{P} b$. Since $1 \in \mathscr{P}$, $P=\mathscr{P} \cap F$, so $0 \leqslant \mathscr{P} a \leqslant \mathscr{P} b$ and by hypothesis we obtain $a \in I_{w}$, so $a \in I_{w} \cap F$.

(2) Let $\varnothing \mathscr{P}:=\pi_{w}\left(\mathscr{P} \cap R_{w}\right)$. We obviously have $\varnothing \mathscr{P} \neq \varnothing, \varnothing \mathscr{P}+\varnothing \mathscr{P} \subseteq \varnothing \mathscr{P}$ and, for $\pi_{w}(a) \in \varnothing \mathscr{P}$ and $\pi_{w}(x) \in A_{0}, \sigma_{0}\left(\pi_{w}(x)\right) \pi_{w}(a) \pi_{w}(x)=\pi_{w}(\sigma(x) a x) \in \varnothing \mathscr{P}$.

We check $\varnothing \mathscr{P} \cap-\varnothing \mathscr{P}=\{0\}$. Let $a \in \mathscr{P} \cap R_{w}$ be such that $\pi_{w}(a) \in \varnothing \mathscr{P} \cap-\varnothing \mathscr{P}$, i.e., there is $b \in \mathscr{P} \cap R_{w}$ such that $\pi_{w}(a)=-\pi_{w}(b)$. Then $a+b \in I_{w}$. By the assumptions on $a$ and $b$ we have $0 \leqslant \mathscr{P} a \leqslant \mathscr{P} a+b$. Therefore, by $\mathscr{P}$-convexity of $I_{w}$, we get $a \in I_{w}$, so $\pi_{w}(a)=0$.

We show that $\varnothing \mathscr{P}$ is over $\bar{P}$, i.e., that $(\varnothing \mathscr{P})_{F_{v}}=\bar{P}$ by showing that $\bar{P} \subseteq$ $(\varnothing \mathscr{P})_{F_{v}}$, cf. Remark $4.2(\overline{\mathscr{P}} \neq\{0\}$ since $\overline{1} \in \overline{\mathscr{P}})$. Let $\pi_{w}(u) \in \bar{P}$ with $u \in$ $P \cap R_{v}=P \cap R_{w}$, and let $\pi_{w}(a) \in \varnothing \mathscr{P}$ with $a \in \mathscr{P} \cap R_{w}$. Then $u a \in \mathscr{P} \cap R_{w}$ by $(\mathrm{P} 4)$ and the definition of gauge, so $\pi_{w}(u a)=\pi_{w}(u) \pi_{w}(a) \in \emptyset \mathscr{P}$.

Note that we will show in Theorem 6.11 that $\pi_{w}\left(\mathscr{P} \cap R_{w}\right)$ is actually maximal, i.e., is a positive cone on $\left(A_{0}, \sigma_{0}\right)$ over $\bar{P}$.

Proposition 6.2. (C5) implies (C4). 
Proof. Let $a \in R_{w}$ and $b \in I_{w}$ be such that $0 \leqslant \mathscr{P} a \leqslant \mathscr{P} b$. So $a \in \mathscr{P}$ and $b=a+c$ for some $c \in \mathscr{P} \cap R_{w}$. Then $0=\pi_{w}(b)=\pi_{w}(a)+\pi_{w}(c)$, therefore $\pi_{w}(a)=-\pi_{w}(c) \in \pi_{w}\left(\mathscr{P} \cap R_{w}\right) \cap-\pi_{w}\left(\mathscr{P} \cap R_{w}\right)=\{0\}$, so $a \in I_{w}$.

Proposition 6.3. (C5) implies that $v$ is compatible with $P$.

Proof. It is sufficient to show that $\pi_{v}\left(P \cap R_{v}\right)$ is an ordering on $F_{v}$, cf. [16, Thm. 3.6.1(1)]. The set $\pi_{v}\left(P \cap R_{v}\right)$ is closed under sum and product and satisfies $F_{v} \subseteq \pi_{v}\left(P \cap R_{v}\right) \cup-\pi_{v}\left(P \cap R_{v}\right)$. Thus to show that $\pi_{v}\left(P \cap R_{v}\right)$ is an ordering on $F_{v}$ we only need to show that it is proper. This follows from the observation that $\mathscr{P} \cap R_{v}=\mathscr{P} \cap F \cap R_{v}=P \cap R_{v}$ and thus $\pi_{v}\left(P \cap R_{v}\right)=\pi_{w}\left(P \cap R_{v}\right) \subseteq \pi_{w}\left(\mathscr{P} \cap R_{w}\right)$, which is proper by the assumption and (P5).

Proposition 6.4. (C6) and (C7) are equivalent.

Proof. Assume that (C6) holds, i.e., $a \in \mathscr{P} \cap I_{w} \Rightarrow 1-a \in \mathscr{P}$ (since $a \neq 1$ ). Let $a \in \operatorname{Sym}\left(I_{w}, \sigma\right)$. Then $a=\sigma(a)$ and, using (P3) and $1 \in \mathscr{P}, a^{2} \in \mathscr{P} \cap I_{w}$. Therefore $1-a^{2} \in \mathscr{P}$. By Proposition 4.13, all $F_{P}$-eigenvalues of $a^{2}$ are at most 1 and so all $F_{P}$-eigenvalues of $a$ are in $[-1,1]$. Thus $1+a$ only has nonnegative $F_{P}$-eigenvalues, and so $1+a \in \mathscr{P}$ by Proposition 4.13 .

Conversely, assume that (C7) holds. Let $a \in \mathscr{P} \cap I_{w}$. Then $a$ and thus $-a$ are in $\operatorname{Sym}\left(I_{w}, \sigma\right)$. Therefore $1-a \in \mathscr{P}$ by the assumption, i.e., $a \leqslant \mathscr{P} 1$. The result follows since $1 \notin I_{w}$.

We now consider the $v_{k, P}$-gauge $w_{k, \mathscr{P}}$, cf. (5.3) in Section 5.2 ,

Proposition 6.5. (C0), .., (C7) hold for $w_{k, \mathscr{P}}$.

Proof. We start by proving (C0), from which (C1) up to (C5) follow.

We first consider the case where $F=F_{P},(A, \sigma)=\left(M_{n}(E),{ }^{-t}\right)$, and $P^{\prime}$ is the unique ordering on $F_{P}$ (with $E$ as in Section 5.1). Let $\mathscr{P}^{\prime}=\operatorname{PSD}_{n}\left(E, P^{\prime}\right)$ and let $a, b \in \mathscr{P}^{\prime}$. Then, for every $x \in E^{n}$, we have $\bar{x}^{t} a x, \bar{x}^{t} b x \in P^{\prime}$. Hence, by the $P^{\prime}$-convexity of $v^{\prime}:=v_{k, P^{\prime}}$

$$
v^{\prime}\left(\bar{x}^{t}(a+b) x\right)=v^{\prime}\left(\bar{x}^{t} a x+\bar{x}^{t} b x\right)=\min \left\{v^{\prime}\left(\bar{x}^{t} a x\right), v^{\prime}\left(\bar{x}^{t} b x\right)\right\} .
$$

Therefore, by Lemma 5.10 (2) (and since all $F_{P}$-eigenvalues are trivially in $F_{P}$ ) for $w^{\prime}:=w_{k, \mathscr{P}^{\prime}}$,

$w^{\prime}(a+b)=\min _{x \in E^{n} \backslash\{0\}}\left\{v^{\prime}\left(\bar{x}^{t}(a+b) x\right)-v^{\prime}\left(\bar{x}^{t} x\right)\right\} \leqslant \min _{x \in E^{n} \backslash\{0\}}\left\{v^{\prime}\left(\bar{x}^{t} a x\right)-v^{\prime}\left(\bar{x}^{t} x\right)\right\}=w^{\prime}(a)$.

Likewise, $w^{\prime}(a+b) \leqslant w^{\prime}(b)$. Therefore, $w^{\prime}(a+b) \leqslant \min \left\{w^{\prime}(a), w^{\prime}(b)\right\}$ and the reverse inequality holds for any value function. The case for general $(A, \sigma)$ then follows easily by extension of scalars to $F_{P}$ and using the map $f_{F_{P}}$, cf. diagram (5.2) at the start of Section 5.2.

Finally, we prove (C6). Then (C7) follows by Proposition 6.4. For $a \in \mathscr{P} \cap$

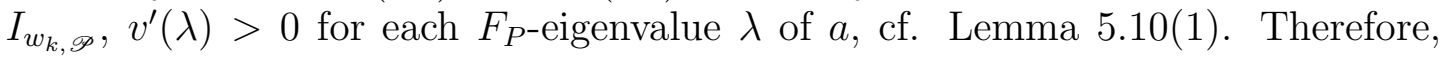


each $F_{P^{-}}$-eigenvalue $1-\lambda$ of $1-a$ satisfies $1-\lambda>_{P^{\prime}} 0$ by convexity of $I_{v^{\prime}}$. Hence, $f_{F_{P}}((1-a) \otimes 1) \in \mathscr{P}^{\prime}$, so $1-a \in \mathscr{P}$.

The fact that $(\mathrm{C} 7)$ holds for $w_{k, \mathscr{P}}$ can be strengthened as follows:

Proposition 6.6. Let $c \in \mathscr{P} \cap R_{w_{k, \mathscr{P}}}$ be such that $\pi_{w_{k, \mathscr{P}}}(c) \in A_{0}^{\times}$, and let $\varepsilon \in$ $\operatorname{Sym}\left(I_{w_{k}, \mathscr{P}}, \sigma\right)$. Then $c+\varepsilon \in \mathscr{P}$.

Proof. (The idea of this proof is due to A. Wadsworth.) We use the notation from the start of Section 5.2. Since $\mathscr{P}=A \cap \mathscr{P}^{\prime}, R_{w_{k, \mathscr{P}}}=A \cap R_{w_{k, \mathscr{P}}}, I_{w_{k, \mathscr{P}}}=$ $A \cap I_{w_{k, \mathscr{P}}}$ and $f_{F_{P}}$ is an isomorphism of algebras with involution, we may assume that $F=F_{P}, P$ is the unique ordering on $F_{P},(A, \sigma)=\left(M_{n}(E),{ }^{t}\right)$ (with $E$ as in Section 5.1), and $\mathscr{P}=\operatorname{PSD}_{n}(E, P)$. Let $v=v_{k, P}$ and $w=w_{k, \mathscr{P}}$.

At the end of the following argument we use that, since $v$ is compatible with $P$, for every $a, b \in F$ with $v(a)>v(b), b>_{P} 0$ implies $a+b>_{P} 0$ (as can easily be checked).

We show $c+\varepsilon \in \operatorname{PSD}_{n}(E, P)$ by showing that $\bar{x}^{t}(c+\varepsilon) x>_{P} 0$ for every $x \in E^{n} \backslash\{0\}$. Observe that, since $I_{w}=\operatorname{ker}\left(\pi_{w}\right)$ is the Jacobson radical of $R_{w}$ (see Remark 3.4), $\pi_{w}(c) \in A_{0}^{\times}$implies $c \in R_{w}^{\times}$. If $\lambda_{1}, \ldots, \lambda_{n}$ are the eigenvalues of $c$, then $\lambda_{1}^{-1}, \ldots, \lambda_{n}^{-1}$ are the eigenvalues of $c^{-1}$, and since both $c$ and $c^{-1}$ are in $R_{w} \backslash I_{w}$, Lemma 5.10(1) yields $v\left(\lambda_{i}\right) \geqslant 0$ and $v\left(\lambda_{i}\right) \leqslant 0$ for every i, i.e., $v\left(\lambda_{1}\right)=$ $\cdots=v\left(\lambda_{n}\right)=0$. In particular, and since $c \in \operatorname{PSD}_{n}(E, P), \lambda_{1}>_{P} 0, \ldots, \lambda_{n}>_{P} 0$.

Let $x \in E^{n} \backslash\{0\}$. Since $w(\varepsilon)>0$ we obtain by Lemma 5.10 (2) that $v\left(\bar{x}^{t} \varepsilon x\right) \geqslant$ $w(\varepsilon)+v\left(\bar{x}^{t} x\right)>v\left(\bar{x}^{t} x\right)$. But, by Lemma 5.10(3), $v\left(\bar{x}^{t} c x\right)=v\left(\bar{x}^{t} x\right)$, so that $v\left(\bar{x}^{t} \varepsilon x\right)>v\left(\bar{x}^{t} c x\right)$. Since $\bar{x}^{t} c x>_{P} 0$ and $v$ is compatible with $P$, it follows that $\bar{x}^{t} c x+\bar{x}^{t} \varepsilon x>_{P}$ 0, i.e., $\bar{x}^{t}(c+\varepsilon) x>_{P} 0$.

Proposition 6.7. ( $\mathrm{C} 0), \ldots,(\mathrm{C} 7)$ are equivalent.

Proof. Since $R_{v}=R_{w} \cap F, I_{v}=I_{w} \cap F$ and $P=\mathscr{P} \cap F$ (since $1 \in \mathscr{P}$ ), each of the properties $(\mathrm{C} 0), \ldots,(\mathrm{C} 7)$ implies the corresponding property where $A$ is replaced by $F, w$ is replaced by $v$, and $\mathscr{P}$ is replaced by $P$. Therefore, any of these properties implies that $v$ is compatible with $P$, i.e., $v=v_{k, P}$ for some subfield $k$ of $F$, cf. [19, Thm. 7.21]. By Theorem [5.16, $w_{k, \mathscr{P}}$ is the unique $\sigma$-invariant $v_{k, P}$-gauge on $(A, \sigma)$ and thus $w=w_{k, \mathscr{P}}$. The other properties then hold since they hold for $w=w_{k, \mathscr{P}}$ by Proposition 6.5.

Definition 6.8. We say that $w$ (recall that $w$ is assumed $\sigma$-invariant) and $\mathscr{P}$ are compatible if any one of the equivalent properties $(\mathrm{C} 0), \ldots,(\mathrm{C} 7)$ holds.

Proposition 6.9. If $w$ is compatible with $\mathscr{P}$, then there exists a subfield $k$ of $F$ such that $v=v_{k, P}$ and $w=w_{k, \mathscr{P}}$. In particular, $w$ is $\sigma$-special.

Proof. As observed in the proof of Proposition 6.7, $v=v_{k, P}$ for some subfield $k$ of $F$. Since $w$ is a $\sigma$-invariant $v$-gauge on $A$, we have $w=w_{k, \mathscr{P}}$ by Theorem 5.16 .

Assume now that $w$ is compatible with $\mathscr{P}$. Let $Q$ be the ordering on $F_{v}$ that is induced by $P$ and let $\mathscr{Q}$ be the unique positive cone on $\left(A_{0}, \sigma_{0}\right)$ that is over $Q$ 
and such that $1 \in \mathscr{Q}$. Observe that $\mathscr{Q}$ exists by property (C5) and Lemma 4.18. We denote by $P^{\prime}$ the unique ordering on $F_{P}$ and by $v^{\prime}$ the natural extension of $v=v_{k, P}$ to $F_{P}$, given by the convex closure of $k$ with respect to $P^{\prime}$, i.e., $v^{\prime}=v_{k, P^{\prime}}$. The following lemma is folklore. We provide a proof for the convenience of the reader.

Lemma 6.10. The residue field $\left(F_{P}\right)_{v^{\prime}}$ is a real closure of $F_{v}$ at $Q$.

Proof. By [10, Thm. 1, p. 66], $\left(F_{P}\right)_{v^{\prime}}$ is real closed. It is also algebraic over $F_{v}$ (since $F_{P}$ is algebraic over $F$ ) and it is immediate that the ordering induced on $\left(F_{P}\right)_{v^{\prime}}$ by $P^{\prime}$ extends the ordering on $F_{v}$ induced by $P$ (i.e., $Q$ ). Therefore $\left(F_{P}\right)_{v^{\prime}}$ is a real closure of $F_{v}$ at $Q$.

In light of Lemma 6.10, we may take $\left(F_{P}\right)_{v^{\prime}}$ as a real closure $\left(F_{v}\right)_{Q}$ of $F_{v}$ at $Q$ in the considerations below. Furthermore, $P^{\prime}$ is then a lifting of the unique ordering $Q^{\prime}$ of $\left(F_{v}\right)_{Q}$.

Theorem 6.11. $\pi_{w}\left(\mathscr{P} \cap R_{w}\right)$ is a positive cone on $\left(A_{0}, \sigma_{0}\right)$ over $Q$ containing 1 .

Proof. By Proposition 6.1 (2) $\pi_{w}\left(\mathscr{P} \cap R_{w}\right)$ is a prepositive cone on $\left(A_{0}, \sigma_{0}\right)$ over $Q$. Let $\mathscr{Q}$ be the unique positive cone on $\left(A_{0}, \sigma_{0}\right)$ over $Q$ containing $\pi_{w}\left(\mathscr{P} \cap R_{w}\right)$, cf. Lemma 4.18, We will show that $\pi_{w}\left(\mathscr{P} \cap R_{w}\right)=\mathscr{Q}$.

We extend scalars to $F_{P}$ and note that by Lemma 4.5, $\left(A \otimes_{F} F_{P}, \sigma \otimes\right.$ id $)$ is a finite-dimensional simple $F_{P}$-algebra with $F_{P}$-linear involution $\sigma \otimes$ id such that $F_{P}=\operatorname{Sym}\left(A \otimes_{F} F_{P}, \sigma \otimes \mathrm{id}\right) \cap Z\left(A \otimes_{F} F_{P}\right)$ (i.e., an $F_{P}$-algebra with involution in the terminology of [4]).

Let $\mathscr{P}^{\prime}$ be the unique positive cone on $\left(A \otimes_{F} F_{P}, \sigma \otimes\right.$ id $)$ over $P^{\prime}$ containing $\mathscr{P}$ (see [4, Prop. 5.8] and the classification of positive cones [4, Thm. 7.5]). We denote the $v^{\prime}$-gauge $w_{k, \mathscr{P}}$ by $w^{\prime}$.

Consider the diagram

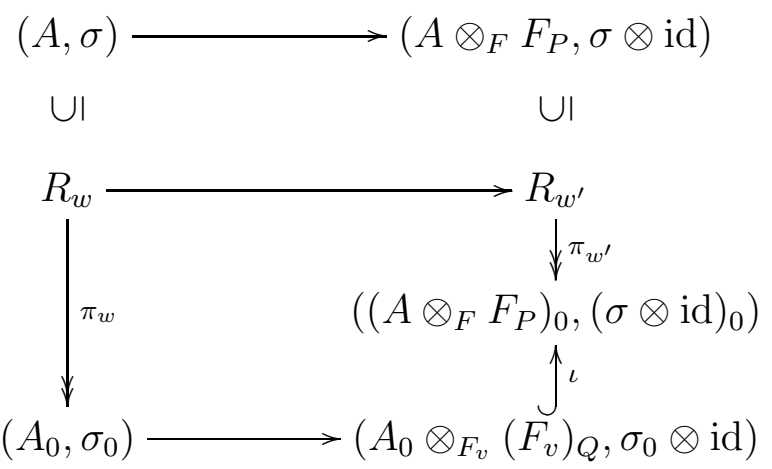

where the horizontal arrows are induced by scalar extension, $\pi_{w^{\prime}}$ denotes the canonical residue map associated to $w^{\prime}$, and $\iota$ denotes the canonical inclusion of $A_{0} \otimes_{F_{v}}\left(F_{v}\right)_{Q}$ in $\left(A \otimes_{F} F_{P}\right)_{0}$, cf. [24, Prop. 2.12], i.e., $\iota: \pi_{w}(x) \otimes \pi_{v^{\prime}}(y) \mapsto \pi_{w^{\prime}}(x \otimes y)$. Note that the lower part of the diagram is commutative by the definition of $\iota$. 
By Proposition 4.14, $\mathscr{P}^{\prime}$ is the set of hermitian squares in $\left(A \otimes_{F} F_{P}, \sigma \otimes \mathrm{id}\right)$. We denote by $\mathscr{Q}^{\prime}$ the unique positive cone on $\left(A_{0} \otimes_{F_{v}}\left(F_{v}\right)_{Q}, \sigma_{0} \otimes\right.$ id $)$ over $Q^{\prime}$ containing $\mathscr{Q}$, and thus 1 (see Proposition 4.6 and Lemma 4.18). By Corollary 4.16, $\mathscr{Q}^{\prime}$ is the set of hermitian squares in $\left(A_{0} \otimes_{F_{v}}\left(F_{v}\right)_{Q}, \sigma_{0} \otimes \mathrm{id}\right)$.

Observe that $\pi_{w^{\prime}}\left(\mathscr{P}^{\prime} \cap R_{w^{\prime}}\right)$ is a prepositive cone on $\left(\left(A \otimes_{F} F_{P}\right)_{0},(\sigma \otimes \mathrm{id})_{0}\right)$ by Proposition 6.5, and thus that $\iota^{-1}\left(\pi_{w^{\prime}}\left(\mathscr{P}^{\prime} \cap R_{w^{\prime}}\right)\right)$ is a prepositive cone over $Q^{\prime}$. Indeed, with reference to Definition 4.1 .

- $\operatorname{properties}(\mathrm{P} 1)$ and $(\mathrm{P} 2)$ are clear (recall that $1 \in \pi_{w^{\prime}}\left(\mathscr{P}^{\prime} \cap R_{w^{\prime}}\right)$ );

- (P3) follows from the fact that $\iota$ is a morphism of algebras with involution and $\pi_{w^{\prime}}\left(\mathscr{P}^{\prime} \cap R_{w^{\prime}}\right)$ is a prepositive cone by property (C5) and Proposition 6.5:

- (P5) holds since $\pi_{w^{\prime}}\left(\mathscr{P}^{\prime} \cap R_{w^{\prime}}\right)$ is a prepositive cone and $\iota$ is injective;

- (P4) holds if the ordering associated to $\iota^{-1}\left(\pi_{w^{\prime}}\left(\mathscr{P}^{\prime} \cap R_{w^{\prime}}\right)\right)$ on $\left(F_{v}\right)_{Q}$ is $Q^{\prime}=\left(\left(F_{v}\right)_{Q}\right)^{2}$. This is the case since it contains $\left(\left(F_{v}\right)_{Q}\right)^{2}$ by $(\mathrm{P} 3)$, and nothing more by $(\mathrm{P} 5)$.

We now show that $\mathscr{Q}^{\prime}=\iota^{-1}\left(\pi_{w^{\prime}}\left(\mathscr{P}^{\prime} \cap R_{w^{\prime}}\right)\right)$. Since $\iota^{-1}\left(\pi_{w^{\prime}}\left(\mathscr{P}^{\prime} \cap R_{w^{\prime}}\right)\right)$ is a prepositive cone over $Q^{\prime}$ and contains 1 , by Lemma 4.18 it is contained in the unique positive cone over $Q^{\prime}$ containing 1 , i.e., in $\mathscr{Q}^{\prime}$.

For the reverse inclusion, let $x \in \mathscr{Q}^{\prime}$. Then $x=\left(\sigma_{0} \otimes \mathrm{id}\right)(y) y$ for some $y \in$ $A_{0} \otimes_{F_{v}}\left(F_{v}\right)_{Q}$. Applying $\iota$ to $x$ and letting $z \in R_{w^{\prime}}$ be such that $\pi_{w^{\prime}}(z)=\iota(y)$, we have $\iota(x)=(\sigma \otimes \mathrm{id})_{0}\left(\pi_{w^{\prime}}(z)\right) \pi_{w^{\prime}}(z)=\pi_{w^{\prime}}((\sigma \otimes \mathrm{id})(z) z)$, which belongs to $\pi_{w^{\prime}}\left(\mathscr{P}^{\prime} \cap R_{w^{\prime}}\right)$ (since $\mathscr{P}^{\prime}$ is the set of hermitian squares in $\left(A \otimes_{F} F_{P}, \sigma \otimes \mathrm{id}\right)$ ). Note that the final equality follows from the definition of the involution $(\sigma \otimes \mathrm{id})_{0}$.

Finally, we show $\mathscr{Q}=\pi_{w}\left(\mathscr{P} \cap R_{w}\right)$. By definition of $\mathscr{Q}$ we have $\pi_{w}\left(\mathscr{P} \cap R_{w}\right) \subseteq$ Q. For the other inclusion we first show that $\mathscr{Q}^{\times} \subseteq \pi_{w}\left(\mathscr{P} \cap R_{w}\right)$. The result will then follow since $\mathscr{Q}=\mathscr{C}\left(\mathscr{Q}^{\times}\right) \subseteq \pi_{w}\left(\mathscr{P} \cap R_{w}\right)$, where the first equality holds by Proposition 4.19 and the inclusion holds by definition of $\mathscr{C}$ since $\pi_{w}\left(\mathscr{P} \cap R_{w}\right)$ is a prepositive cone.

Let $a=\pi_{w}(b) \in \mathscr{Q}^{\times}$with $b \in R_{w} \backslash I_{w}$. We first check that we may assume that $b$ is symmetric: Since $a$ is symmetric, $\sigma_{0}\left(\pi_{w}(b)\right)=\pi_{w}(b)$, i.e., $\pi_{w}(\sigma(b))=\pi_{w}(b)$. Therefore, $b-\sigma(b) \in I_{w}$ and it follows that $\pi_{w}(b)=\pi_{w}\left(\frac{1}{2}(b+\sigma(b))\right)$.

The lower part of diagram (6.1) then yields diagram (6.2):

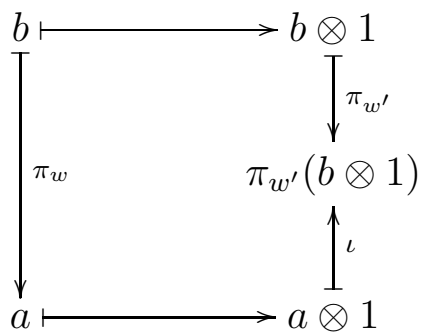


Observe that $a \otimes 1 \in \mathscr{Q}^{\prime \times}$ and so $\iota(a \otimes 1)=\pi_{w^{\prime}}(c)$ for some $c \in \mathscr{P}^{\prime} \cap R_{w^{\prime}}$ since $\mathscr{Q}^{\prime}=\iota^{-1}\left(\pi_{w^{\prime}}\left(\mathscr{P}^{\prime} \cap R_{w^{\prime}}\right)\right)$, and $\pi_{w^{\prime}}(c)$ is invertible.

The commutativity of diagram (6.2) then implies that $\pi_{w^{\prime}}(b \otimes 1)=\pi_{w^{\prime}}(c)$, so $b \otimes 1=c+\varepsilon$ for some $\varepsilon \in \operatorname{Sym}\left(I_{w^{\prime}}, \sigma \otimes \mathrm{id}\right)$. Thus, since $c \in \mathscr{P}^{\prime}$ and by Proposition 6.6, we obtain $b \otimes 1 \in \mathscr{P}^{\prime}$ and so $b \in \mathscr{P}$.

Remark 6.12. Observe that by Theorem 6.11, we can replace (C5) by $\left(\mathrm{C} 5^{\prime}\right) \pi_{w}\left(\mathscr{P} \cap R_{w}\right)$ is a positive cone on $\left(A_{0}, \sigma_{0}\right)$.

\section{Strong ANisotropy of RESIDUALly POSITIVE FORMS}

In this section we collect a number of technical results for use in Section 8 . Let $A$ be a finite-dimensional semisimple $F$-algebra with $F$-linear involution $\sigma$.

Let $w: A \rightarrow \Gamma \cup\{\infty\}$ be a $v$-gauge on $A$ and denote the canonical projection $R_{w} \rightarrow A_{0}$ by $\pi_{w}$. For each $n \in \mathbb{N}$ we define

$$
w_{n}: M_{n}(A) \rightarrow \Gamma \cup\{\infty\}, w_{n}\left(\left(a_{i j}\right)_{i, j}\right):=\min _{i, j}\left\{w\left(a_{i j}\right)\right\} .
$$

A straightforward computation shows that $w_{n}$ is a surmultiplicative $v$-value function. We denote the canonical projection $R_{w_{n}} \rightarrow\left(M_{n}(A)\right)_{0}$ by $\pi_{w_{n}}$.

Using the definition of $w_{n}$, it is easy to check that $R_{w_{n}}=M_{n}\left(R_{w}\right), I_{w_{n}}=M_{n}\left(I_{w}\right)$ and that the map

$$
\xi:\left(M_{n}(A)\right)_{0} \rightarrow M_{n}\left(A_{0}\right), \pi_{w_{n}}\left(\left(a_{i j}\right)_{i, j}\right) \mapsto\left(\pi_{w}\left(a_{i j}\right)\right)_{i, j}
$$

is an isomorphism of $F_{v}$-algebras.

Assume that $w$ is invariant under $\sigma$. Let $U=\operatorname{diag}\left(u_{1}, \ldots, u_{n}\right)$ with $u_{i} \in R_{v}^{\times}=$ $R_{v} \backslash I_{v}$. Then

$$
\left(\operatorname{Int}(U) \circ \sigma^{t}\right)\left(\left(a_{i j}\right)_{i, j}\right)=\left(u_{i} u_{j}^{-1} \sigma\left(a_{j i}\right)\right)_{i, j}
$$

and so

$$
\begin{aligned}
w_{n}\left(\left(\operatorname{Int}(U) \circ \sigma^{t}\right)\left(\left(a_{i j}\right)_{i, j}\right)\right) & =\min _{i, j}\left\{w\left(u_{i} u_{j}^{-1} \sigma\left(a_{j i}\right)\right)\right\} \\
& =\min _{i, j}\left\{w\left(a_{j i}\right)+v\left(u_{i}\right)-v\left(u_{j}\right)\right\} \\
& =\min _{i, j}\left\{w\left(a_{j i}\right)\right\} \\
& =w_{n}\left(\left(a_{i j}\right)_{i, j}\right),
\end{aligned}
$$

i.e., $w_{n}$ is invariant under $\operatorname{Int}(U) \circ \sigma^{t}$. It follows that $\operatorname{Int}(U) \circ \sigma^{t}$ induces a gradepreserving involution on $\operatorname{gr}_{w_{n}}\left(M_{n}(A)\right)$.

Lemma 7.1. The map $\xi$ is an isomorphism from $\left(\left(M_{n}(A)\right)_{0},\left(\operatorname{Int}(U) \circ \sigma^{t}\right)_{0}\right)$ to $\left(M_{n}\left(A_{0}\right), \operatorname{Int}\left(\xi \circ \pi_{w_{n}}(U)\right) \circ\left(\sigma_{0}\right)^{t}\right)$.

Proof. Since $u_{1}, \ldots, u_{n} \in R_{v} \backslash I_{v}$, a simple computation shows that

$$
\xi\left(\pi_{w_{n}}\left(\left(\operatorname{Int}(U) \circ \sigma^{t}\right)(X)\right)\right)=\operatorname{Int}\left(\pi_{w_{n}}(U)\right) \circ\left(\sigma_{0}\right)^{t}\left(\xi\left(\pi_{w_{n}}(X)\right)\right)
$$


for all $X \in R_{w_{n}}=M_{n}\left(R_{w}\right)$, i.e.,

$$
\xi\left(\left(\operatorname{Int}(U) \circ \sigma^{t}\right)_{0}\left(\pi_{w_{n}}(X)\right)\right)=\operatorname{Int}\left(\pi_{w_{n}}(U)\right) \circ\left(\sigma_{0}\right)^{t}\left(\xi\left(\pi_{w_{n}}(X)\right)\right)
$$

for all $X \in R_{w_{n}}=M_{n}\left(R_{w}\right)$, proving the result.

Let $R$ be a semisimple ring and let $\tau$ be an involution on $R$. We say that $\tau$ is anisotropic if there is no nonzero $x \in R$ such that $\tau(x) x=0$ and, for $r \in \mathbb{N}$, that $\tau$ is $r$-anisotropic if the involution $\tau^{t}$ on $M_{r}(R)$ is anisotropic. Furthermore, we say that $\tau$ is strongly anisotropic if $\tau$ is $r$-anisotropic for every $r \in \mathbb{N}$.

Let $M$ be a right $R$-module, let $h: M \times M \rightarrow R$ be a nonsingular hermitian form over $(R, \tau)$ and let $\operatorname{ad}_{h}$ be the adjoint involution of $h$ on $\operatorname{End}_{R}(M)$. Recall that $h\left(\operatorname{ad}_{h}(f)(x), y\right)=h(x, f(y))$ for all $x, y \in M$ and $f \in \operatorname{End}_{R}(M)$. We call $h$ $r$-anisotropic if $r \times h$ is anisotropic.

Lemma 7.2. $h$ is anisotropic if and only if $\operatorname{ad}_{h}$ is anisotropic.

Proof. The left to right implication is straightforward. We prove the other direction by contraposition. Assume that $h$ is isotropic, i.e., assume that there is an $x \in M, x \neq 0$, such that $h(x, x)=0$. Consider the submodule $x R$ of $M$. Since $R$ is semisimple, $x R$ is a direct summand of $M$. Let $f$ be the projection from $M$ to $x R$. Then $\operatorname{ad}_{h}(f) f=0$. Indeed, for all $y, z \in M$ we have

$$
h\left(\operatorname{ad}_{h}(f) f(y), z\right)=h(f(y), f(z))=0
$$

since $h(x, x)=0$. Since $h$ is nonsingular we must have $\operatorname{ad}_{h}(f) f(y)=0$ for all $y \in M$.

Lemma 7.3. Let $r \in \mathbb{N}$. There is an isomorphism of rings with involution

$$
\left(\operatorname{End}_{R}\left(M^{r}\right), \operatorname{ad}_{r \times h}\right) \cong\left(M_{r}\left(\operatorname{End}_{R}(M)\right),\left(\operatorname{ad}_{h}\right)^{t}\right),
$$

where $r \times h:=h \perp \cdots \perp h(r$ times $)$.

Proof. Write $M^{r}=M_{1} \times \cdots \times M_{r}$, where $M_{1}=\cdots=M_{r}=M$. Consider the ring isomorphism

$$
\mu: \operatorname{End}_{R}\left(M^{r}\right) \rightarrow M_{r}\left(\operatorname{End}_{R}(M)\right), f \mapsto\left(f_{i j}\right)_{i, j},
$$

where $\left.f\right|_{M_{i}}=\left(f_{1 i}, \ldots, f_{r i}\right)$ with $f_{i j}: M_{j} \rightarrow M_{i}$, cf. [8, Chap. 9, Prop. 2.3]. A lengthy, but straightforward computation then shows that $\left(\operatorname{ad}_{h}\right)^{t} \circ \mu=\mu \circ$ $\operatorname{ad}_{r \times h}$.

Lemmas 7.2 and 7.3 have been proved for general nonsingular hermitian forms, but will only be used in Lemma 7.4 for a nonsingular diagonal hermitian form, in which case their proofs can be obtained by a simple computation.

Lemma 7.4. Let $s_{1}, \ldots, s_{n} \in \operatorname{Sym}(R, \tau) \cap R^{\times}$and $S=\operatorname{diag}\left(s_{1}, \ldots, s_{n}\right)$. Then for any $r \in \mathbb{N}$, $\operatorname{Int}(S) \circ \tau^{t}$ is $r$-anisotropic on $M_{n}(R)$ if and only if the hermitian form $\left\langle s_{1}, \ldots, s_{n}\right\rangle_{\tau}$ is $r$-anisotropic. 
Proof. Observe that $\operatorname{Int}(S) \circ \tau^{t}$ is adjoint to the hermitian form $\left\langle s_{1}^{-1}, \ldots, s_{n}^{-1}\right\rangle_{\tau}$. Therefore, $\operatorname{Int}(S) \circ \tau^{t}$ is $r$-anisotropic on $M_{n}(R)$ if and only if $\left(\operatorname{Int}(S) \circ \tau^{t}\right)^{t}$ is anisotropic on $M_{r}\left(M_{n}(R)\right)$ if and only if $\operatorname{ad}_{r \times\left\langle s_{1}^{-1}, \ldots, s_{n}^{-1}\right\rangle_{\tau}}$ is anisotropic on $M_{r n}(R)$ by Lemma 7.3 if and only if $r \times\left\langle s_{1}^{-1}, \ldots, s_{n}^{-1}\right\rangle_{\tau}$ is anisotropic by Lemma 7.2. The result follows since the forms $\left\langle s_{1}^{-1}, \ldots, s_{n}^{-1}\right\rangle_{\tau}$ and $\left\langle s_{1}, \ldots, s_{n}\right\rangle_{\tau}$ are isometric.

Recall from Remark 3.3 that the existence of a $\sigma$-special gauge implies that $\sigma$ is anisotropic. This observation will be used for several involutions in what follows.

Proposition 7.5. Let $n \in \mathbb{N}$. Then:

(1) $w_{n}$ is a v-gauge on $M_{n}(A)$.

(2) If the involution $\sigma_{0}$ on $A_{0}$ is n-anisotropic, then $w_{n}$ is $\sigma^{t}$-special.

Proof. (1) Let $\left\{a_{\ell}\right\}$ be a splitting basis of $A$ with respect to $w$ and let $\left\{E_{i j}\right\}$ denote the standard basis of $M_{n}(F)$. Then an easy computation shows that $\left\{a_{\ell} E_{i j}\right\}$ is a splitting basis of $M_{n}(A)$ with respect to $w_{n}$, thus $w_{n}$ is a $v$-norm. The canonical map

$$
\left(M_{n}(A)\right)_{\gamma} \rightarrow M_{n}\left(A_{\gamma}\right),\left(a_{i j}\right)_{i, j}+\left(M_{n}(A)\right)_{>\gamma} \mapsto\left(a_{i j}+A_{>\gamma}\right)_{i, j}
$$

induces an isomorphism of the graded algebras $\operatorname{gr}_{w_{n}}\left(M_{n}(A)\right)$ and $M_{n}\left(\operatorname{gr}_{w}(A)\right)$, and since the latter is semisimple (writing an ideal $I$ of $M_{n}\left(\operatorname{gr}_{w}(A)\right)$ as $M_{n}(J)$ for some ideal $J$ of $\operatorname{gr}_{w}(A)$, a direct verification show that $M_{n}\left(\operatorname{gr}_{w}(A)\right)$ does not contain any nontrivial 2-sided nilpotent homogeneous ideal since $\operatorname{gr}_{w}(A)$ does not), the former is also semisimple. We conclude that $w_{n}$ is a $v$-gauge on $M_{n}(A)$.

(2) The indices 0 below are with respect to the gradings induced by $w$ and $w_{n}$, as appropriate. We follow the arguments in the proof of [13, Prop. 2.12 (iii) $\Rightarrow(\mathrm{i})$ ] (without the restriction that $\sigma$ is of the first kind and noting that the assumption that $j$ is a norm appears to be missing): Since $\sigma_{0}$ is $n$-anisotropic, $\left(\sigma_{0}\right)^{t}$ is anisotropic on $M_{n}\left(A_{0}\right)$. By Lemma 7.1 with $U=I_{n}$ we have $\left(\left(M_{n}(A)\right)_{0},\left(\sigma^{t}\right)_{0}\right) \cong$ $\left(M_{n}\left(A_{0}\right),\left(\sigma_{0}\right)^{t}\right)$ and so we obtain that $\left(\sigma^{t}\right)_{0}$ is anisotropic on $\left(M_{n}(A)\right)_{0}$. Since $w$ is $\sigma$-invariant, $w_{n}$ is $\sigma^{t}$-invariant and the result follows from [23, Prop. 1.1 $(\mathrm{b}) \Rightarrow(\mathrm{a})]$.

Proposition 7.6. Assume that there is a positive cone $\mathscr{Q}$ on $\left(A_{0}, \sigma_{0}\right)$ over $Q \in$ $X_{F_{v}}$ with $1 \in \mathscr{Q}$. Then for any $\ell \in \mathbb{N}$ and any $u_{1}, \ldots, u_{\ell} \in R_{v}^{\times}=R_{v} \backslash I_{v}$ such that $\pi_{v}\left(u_{i}\right) \in Q$, the hermitian form $\left\langle u_{1}, \ldots, u_{\ell}\right\rangle_{\sigma}$ is strongly anisotropic.

Proof. We first show that for all $\ell \in \mathbb{N}$ and $u_{1}, \ldots, u_{\ell} \in R_{v} \backslash I_{v}$ with $\pi_{v}\left(u_{i}\right) \in Q$, the hermitian form $\left\langle\pi_{v}\left(u_{1}\right), \ldots, \pi_{v}\left(u_{\ell}\right)\right\rangle_{\sigma_{0}}$ is anisotropic over $\left(A_{0}, \sigma_{0}\right)$. Assume therefore that there exist $x_{1}, \ldots, x_{\ell} \in R_{w}$ such that $\sum_{i=1}^{\ell} \pi_{v}\left(u_{i}\right) \sigma_{0}\left(\pi_{w}\left(x_{i}\right)\right) \pi_{w}\left(x_{i}\right)=0$. Then, by Lemma 4.17, every $\pi_{v}\left(u_{i}\right) \sigma_{0}\left(\pi_{w}\left(x_{i}\right)\right) \pi_{w}\left(x_{i}\right)$ is zero, and since $\pi_{v}\left(u_{i}\right) \neq 0$ it follows that $\sigma_{0}\left(\pi_{w}\left(x_{i}\right)\right) \pi_{w}\left(x_{i}\right)=0$ for every $i$. Therefore, $\pi_{w}\left(x_{i}\right)=0$ for every $i$ by Lemma 4.17 and since $1 \in \mathscr{Q}$. Since there is no bound on $\ell$, it follows that $\left\langle\pi_{v}\left(u_{1}\right), \ldots, \pi_{v}\left(u_{\ell}\right)\right\rangle_{\sigma_{0}}$ is strongly anisotropic over $\left(A_{0}, \sigma_{0}\right)$.

We now consider the gauge $w_{\ell}$ on $M_{\ell}(A)$. By Lemma [7.4, and with $S:=$ $\operatorname{diag}\left(u_{1}, \ldots, u_{\ell}\right)$, the involution $\operatorname{Int}\left(\xi \circ \pi_{w_{\ell}}(S)\right) \circ \sigma_{0}^{t}$ is strongly anisotropic on $M_{\ell}\left(A_{0}\right)$ 
and so $\left(\operatorname{Int}(S) \circ \sigma^{t}\right)_{0}$ is strongly anisotropic on $\left(M_{\ell}(A)\right)_{0}$ by Lemma 7.1. Then by Proposition 7.5(2) with $n=1$ (applied to $\left(M_{\ell}(A), \operatorname{Int}(S) \circ \sigma^{t}\right)$ and $w_{\ell}$ instead of $(A, \sigma)$ and $w)$, the gauge $w_{\ell}$ is $\operatorname{Int}(S) \circ \sigma^{t}$-special, and thus $\operatorname{Int}(S) \circ \sigma^{t}$ is anisotropic on $M_{\ell}(A)$ by Remark 3.3. Therefore, the form $\left\langle u_{1}, \ldots, u_{\ell}\right\rangle_{\sigma}$ is anisotropic by Lemma 7.4, and thus strongly anisotropic since there is no bound on $\ell$.

Lemma 7.7. Assume that $\Gamma_{w}=\Gamma_{v}$. Let $\omega \in F^{\times}$be such that $v(\omega) \notin 2 \Gamma_{v}$ and let $v^{\prime}$ be the unique extension of $v$ to $F(\sqrt{\omega})$. Then, using the (tame) $v^{\prime}$-gauge $w \otimes v^{\prime}$ on $A \otimes_{F} F(\sqrt{\omega})$, we have

$$
\left(A_{0}, \sigma_{0}\right) \cong\left(\left(A \otimes_{F} F(\sqrt{\omega})\right)_{0},(\sigma \otimes \mathrm{id})_{0}\right)
$$

as $F_{v}$-algebras with involution.

Proof. By hypothesis $\Gamma_{w} \cap \Gamma_{v^{\prime}}=\Gamma_{v}$, so by [24, Prop. 2.12], the map

$$
A_{0} \otimes_{F_{0}} F(\sqrt{\omega})_{0} \rightarrow\left(A \otimes_{F} F(\sqrt{\omega})\right)_{0}, \quad \pi_{w}(a) \otimes \pi_{v^{\prime}}(z) \mapsto \pi_{w \otimes v^{\prime}}(a \otimes z)
$$

is an isomorphism of $F_{0}$-vector spaces. A direct verification using the definition of the product in the graded algebras and the definition of the residue involution shows that it is a morphism of algebras with involution.

Again, a direct verification shows that the $F_{0}$-algebras with involution $\left(A_{0} \otimes_{F_{0}}\right.$ $\left.F(\sqrt{\omega})_{0}, \sigma_{0} \otimes \mathrm{id}\right)$ and $\left(A_{0}, \sigma_{0}\right)$ are isomorphic via the canonical map, and the result follows.

Proposition 7.8. Assume that $\Gamma_{w}=\Gamma_{v}$ and that there is a positive cone $\mathscr{Q}$ on $\left(A_{0}, \sigma_{0}\right)$ over $Q \in X_{F_{v}}$ with $1 \in \mathscr{Q}$. Let $P \in X_{F}$ be a lifting of $Q$ to $F$ (i.e., $\pi_{v}\left(P \cap R_{v}\right)=Q$ ) and let $a_{1}, \ldots, a_{\ell} \in P \backslash\{0\}$. Then the hermitian form $\left\langle a_{1}, \ldots, a_{\ell}\right\rangle_{\sigma}$ is strongly anisotropic.

Proof. Since $a_{1}, \ldots, a_{\ell} \in F$, we can write

$$
\left\langle a_{1}, \ldots, a_{\ell}\right\rangle_{\sigma} \simeq \rho_{1} h_{1} \perp \cdots \perp \rho_{s} h_{s},
$$

where $v\left(\rho_{1}\right), \ldots, v\left(\rho_{r}\right)$ are all different in $\Gamma_{v} / 2 \Gamma_{v}$ and $h_{i}=\left\langle u_{i, 1}, \ldots, u_{i, k_{i}}\right\rangle_{\sigma}$ with $v\left(u_{i, j}\right)=0$ for all $i, j$. Let $\omega_{1}, \ldots, \omega_{r} \in F$ be such that $v\left(\omega_{1}\right), \ldots, v\left(\omega_{r}\right)$ form a $\mathbb{Z} / 2 \mathbb{Z}$-basis of the subspace of $\Gamma_{v} / 2 \Gamma_{v}$ generated by $v\left(\rho_{1}\right), \ldots, v\left(\rho_{s}\right)$. Up to replacing $\omega_{i}$ by $-\omega_{i}$ we can assume that $\omega_{1}, \ldots, \omega_{r} \in P$.

We consider $L:=F\left(\sqrt{\omega_{1}}, \ldots, \sqrt{\omega_{r}}\right)$. Since the elements $v\left(\omega_{i}\right)$ are linearly independent in $\Gamma_{v} / 2 \Gamma_{v}$, there is only one extension $v^{\prime}$ of $v$ to $L$ and $F_{v}=L_{v^{\prime}}$, cf. [6, Thm. 3.3.4]. Moreover, $P$ extends to an ordering $P^{\prime}$ on $L$, and $v^{\prime}$ is compatible with $P^{\prime}$ (indeed: write $v$ as $v_{k, P}$; then $v_{k, P^{\prime}}$ extends $v$ to $L$, so is equal to $\left.v^{\prime}\right)$. By definition of $L, v^{\prime}\left(a_{i}\right) \in 2 \Gamma_{v^{\prime}}$ for $i=1, \ldots, \ell$, so that $a_{i}=u_{i} b_{i}^{2}$ for some $u_{i}, b_{i} \in L$ with $v^{\prime}\left(u_{i}\right)=0$. Therefore

$$
\left\langle a_{1}, \ldots, a_{\ell}\right\rangle_{\sigma \otimes \mathrm{id}} \simeq\left\langle u_{1}, \ldots, u_{\ell}\right\rangle_{\sigma \otimes \mathrm{id}}
$$

over $\left(A \otimes_{F} L, \sigma \otimes \mathrm{id}\right)$, and $u_{i}=a_{i} b_{i}^{-2} \in P^{\prime}$, so $\pi_{v^{\prime}}\left(u_{i}\right) \in Q$ for $i=1, \ldots, \ell$.

Furthermore, by applying Lemma $7.7 r$ times, we see that $\left(\left(A \otimes_{F} L\right)_{0},(\sigma \otimes\right.$ id $\left.)_{0}\right) \cong\left(A_{0}, \sigma_{0}\right)$, so that there is a positive cone containing 1 on $\left(\left(A \otimes_{F} L\right)_{0},(\sigma \otimes\right.$ 
id) $)_{0}$ ) over $Q$. Since $A \otimes_{F} L$ is semisimple and $\sigma \otimes$ id is $L$-linear, we can apply Proposition 7.6 with $A \otimes_{F} L$, and obtain that $\left\langle a_{1}, \ldots, a_{\ell}\right\rangle_{\sigma \otimes \text { id }}$ is strongly anisotropic. The result follows.

\section{Baer-Krull type Results For Positive CONes AND Gauges}

Let $v: F \rightarrow \Gamma_{v} \cup\{\infty\}$ be a valuation on $F$. We recall the following presentation of the classical Baer-Krull theorem, cf. [20, pp. 27-28]: Let $\Omega:=\left\{\omega_{i}\right\}_{i \in I} \subseteq F^{\times}$ be such that $\left\{v\left(\omega_{i}\right)\right\}_{i \in I}$ is a $\mathbb{Z} / 2 \mathbb{Z}$-basis of $\Gamma_{v} / 2 \Gamma_{v}$ and let $\Omega_{\text {prod }}$ be the set of all finite products of elements of $\Omega$ (including 1). Each $a \in F$ can be written in the form $a=u b^{2} \rho$ with $v(u)=0, b \in F$ and $\rho \in \Omega_{\text {prod }}$.

Theorem 8.1 (Baer-Krull). Let $\eta: \Omega \rightarrow\{-1,1\}$ be any map, extended multi-

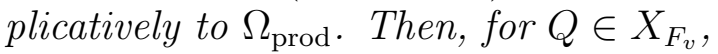

$$
P_{(\eta, Q)}:=\left\{a=u b^{2} \rho \in F \mid \pi_{v}(u) \cdot \eta(\rho) \in Q\right\}
$$

is an ordering on $F$ that is a lifting of $Q$ (i.e., $\pi_{v}\left(P_{(\eta, Q)} \cap R_{v}\right)=Q$ ), and all the liftings of $Q$ are of this form. In this case, $v$ is compatible with $P_{(\eta, Q)}$.

Assume now that $A$ is a finite-dimensional simple $F$-algebra with $F$-linear involution $\sigma$ and centre $K=Z(A)$ such that $F=\operatorname{Sym}(A, \sigma) \cap K$. Let $w$ be a $\sigma$-invariant $v$-gauge on $A$ and let $\mathscr{Q}$ be a positive cone on $\left(A_{0}, \sigma_{0}\right)$ over $Q \in X_{F_{v}}$, such that $1 \in \mathscr{Q}$. By Theorem 8.1 , if $P$ is a lifting of $Q$, then $v$ is compatible with $P$ and so $v=v_{k, P}$ for some subfield $k$ of $F$, cf. [19, Thm. 7.21].

A positive cone $\mathscr{P}$ on $(A, \sigma)$ over $P$ is called a lifting of $\mathscr{Q}$ (with respect to $w$ ) if $P$ is a lifting of $Q$ with respect to $v$ and

$$
\pi_{w}\left(\mathscr{P} \cap R_{w}\right)=\mathscr{Q} .
$$

We consider the involution trace form

$$
T_{(A, \sigma)}: A \times A \rightarrow K,(x, y) \mapsto \operatorname{Trd}_{A}(\sigma(x) y),
$$

which is a symmetric bilinear form over $F$ if $\sigma$ is of the first kind and a hermitian form over $(K, \iota)$ if $\sigma$ is of the second kind, where $\iota$ is the nontrivial $F$-automorphism of $K$. Note that $T_{(A, \sigma)}$ is nondegenerate, cf. [12, §11]. Let $\alpha_{1}, \ldots, \alpha_{m} \in F$ be the entries in a diagonalization of $T_{(A, \sigma)}$, where $m=\operatorname{dim}_{K} A$.

Theorem 8.2. Let $P \in X_{F}$. The following statements are equivalent:

(1) $\mathscr{Q}$ lifts to a positive cone $\mathscr{P}$ on $(A, \sigma)$ over $P$, that contains 1 . In this case $w=w_{k, \mathscr{P}}\left(\right.$ where $\left.v=v_{k, \mathscr{P}}\right)$ and in particular is compatible with $\mathscr{P}$.

(2) $\sigma$ is positive at $P$.

(3) The form $T_{(A, \sigma)}$ is definite at $P$.

(4) $\alpha_{1}, \ldots, \alpha_{m} \in P$ or $\alpha_{1}, \ldots, \alpha_{m} \in-P$.

Proof. The equivalence $(2) \Leftrightarrow(3)$ is [3, Prop. 4.8] with $u=1$, and the equivalence $(3) \Leftrightarrow(4)$ is clear.

$(1) \Rightarrow(2)$ : This follows from [4, Cor. 7.7]. 
$(2) \Rightarrow(1)$ : Let $k$ be a subfield of $F$ such that $v=v_{k, P}$. By [4, Cor. 7.7], there exists a positive cone $\mathscr{P}$ on $(A, \sigma)$ over $P$ such that $1 \in \mathscr{P}$. By Theorem 5.16 and Proposition 3.8, $w_{k, \mathscr{P}}$ is $\sigma$-special and thus the unique $\sigma$-invariant $v$-gauge on $A$, so $w=w_{k, \mathscr{P}}$. Therefore, $w$ and $\mathscr{P}$ are compatible by Proposition 6.5, and we conclude with Theorem 6.11 and Lemma 4.18 .

The equivalent conditions of Theorem 8.2 are not always satisfied despite the existence of the positive cone $\mathscr{Q}$, as the following example shows.

Example 8.3. Let $F=\mathbb{R}((x))((y))$ be the iterated Laurent series field in the unknowns $x$ and $y$ and let $v: F \rightarrow \mathbb{Z} \times \mathbb{Z}$ be the standard $(x, y)$-adic (Henselian) valuation on $F$ (cf. [25, §3]). Note that $F$ has exactly four orderings, determined by the signs of $x$ and $y$.

Let $A=(x, y)_{F}$ be the quaternion division algebra with generators $i$ and $j$ such that $i^{2}=x$ and $j^{2}=y$. Let $\gamma$ be the canonical symplectic involution (quaternion conjugation) on $A$ and also consider the orthogonal involution $\sigma=\operatorname{Int}(i) \circ \gamma$ on A. Straightforward computations (or see [12, (11.3)(1)]) show that

$$
T_{(A, \gamma)} \simeq\langle 2,-2 x,-2 y, 2 x y\rangle \text { and } T_{(A, \sigma)} \simeq\langle 2,-2 x, 2 y,-2 x y\rangle .
$$

Since $v$ is Henselian it extends uniquely to a valuation $w$ on $A$ (cf. [17, Thm. 2]), specifically $w(a)=\frac{1}{2} v(\gamma(a) a)$, cf. [25, (2.7)]. Since char $F_{v}=0, w$ is a $v$-gauge on $A$ by [22, Prop. 1.13]. Observe that any $F$-linear involution $\tau$ on $A$ must be anisotropic since $A$ is a division algebra. With reference to [2, Ex. 6.8] we note that $A_{0}=F_{v}=\mathbb{R}$.

Let $S$ be the ordering on $F$ where $x<0$ and $y<0$. Then $A \otimes_{F} F_{S} \cong(-1,-1)_{F_{S}}$, and so $S \in \operatorname{Nil}[A, \sigma]$ and $S \notin \operatorname{Nil}[A, \gamma]$, cf. [1, Def. 3.7]. If $P$ is any of the other three orderings on $F$, then $A \otimes_{F} F_{P} \cong M_{2}\left(F_{P}\right)$, and so $P \notin \operatorname{Nil}[A, \sigma]$, but $P \in \operatorname{Nil}[A, \gamma]$. Therefore, $\operatorname{Nil}[A, \sigma]=\{S\}$ and $\operatorname{Nil}[A, \gamma]=X_{F} \backslash\{S\}$.

Note that since $A_{0}=\mathbb{R}, \gamma_{0}=\sigma_{0}=\mathrm{id}$. Therefore, the only positive cone on $\left(A_{0}, \gamma_{0}\right)=\left(A_{0}, \sigma_{0}\right)=(\mathbb{R}$, id $)$ that contains 1 is the unique ordering $Q$ of $\mathbb{R}$.

Theorem 8.2 describes the orderings of $F$ for which this positive cone can be lifted to a positive cone that contains 1 . In case of $(A, \gamma)$ this is precisely $S$. In case of $(A, \sigma)$ it is the unique ordering with $x<0$ and $y>0$.

Observe that for $(A, \gamma)$ the orderings for which we cannot lift the positive cone $Q$ are exactly the orderings in $\operatorname{Nil}[A, \gamma]$, but that the corresponding set for $(A, \sigma)$ contains both orderings that are in $\operatorname{Nil}[A, \sigma]$ and orderings that are not. In the latter case observe that there is a positive cone on $(A, \sigma)$ over the ordering, but it will not contain 1, cf. [4, Prop. 6.6].

In view of Theorem 8.2 and Example 8.3 , if $P \in X_{F}$ is a lifting of $Q$, there may not always be a lifting $\mathscr{P}$ of $\mathscr{Q}$ over $P$ that contains 1 . In order to describe when such liftings always exists, we investigate more closely the behaviour of $T_{(A, \sigma)}$.

We define

$$
\operatorname{Lift}(\mathscr{Q}):=\left\{P \in X_{F} \mid \text { there is a lifting of } \mathscr{Q} \text { over } P \text {, that contains } 1\right\} .
$$


Let $\left({ }^{\iota} A,{ }^{\iota} \sigma\right)$ denote the conjugate algebra with involution of $(A, \sigma)$, cf. [12, $\S 3 . \mathrm{B}]$. There is an isomorphism of $K$-algebras with involution

$$
\left(A \otimes_{K} \iota A, \sigma \otimes{ }^{\iota} \sigma\right) \stackrel{\sim}{\longrightarrow}\left(\operatorname{End}_{K}(A), \operatorname{ad}_{T_{(A, \sigma)}}\right),
$$

cf. [12, Prop. 11.1]. Clearly, the $v$-gauge $w$ is defined on ${ }^{\iota} A$ as well: $w\left({ }^{\iota} a\right):=w(a)$ for all $a \in A$. Let $v^{\prime}$ be an extension of $v$ to $K$. Recall that $\Gamma_{v^{\prime}}=\Gamma_{v} \cup\left(\frac{1}{2} v(d)+\Gamma_{v}\right)$, where $K=F(\sqrt{d})$.

Lemma 8.4. Assume that $\sigma$ is of the second kind, and let $P \in \operatorname{Lift}(\mathscr{Q})$. Then $P$ does not extend to $K=Z(A)$.

Proof. If $P$ extends to $Z(A)$, then $P \in \operatorname{Nil}[A, \sigma]$ by [4, Prop. 8.4]. Indeed, Nil $[A, \sigma]$ only depends on the Brauer class of $A$ and the type of $\sigma$, cf. [1, Def. 3.7]. Let $D$ be the skewfield part of $A$. Then $D$ also carries an involution of the second kind (cf. [12, Thm. 3.1]), which we call $\vartheta$. Then $\operatorname{Nil}[A, \sigma]=\operatorname{Nil}[D, \vartheta]$ and we can apply [4, Prop. 8.4] since $Z(A)=Z(D)$.

This contradicts that $\sigma$ is positive at $P$ (cf. Theorem [8.2) by [4, Thm. 6.8].

Lemma 8.5. Assume that $\operatorname{Lift}(\mathscr{Q}) \neq \varnothing$. Then

(1) $v^{\prime}$ is the unique extension of $v$ to $K$;

(2) $w$ is a $v^{\prime}$-gauge on $A$;

(3) $w \otimes w$ is a $\sigma \otimes{ }^{\iota} \sigma$-invariant $v^{\prime}$-gauge and $v$-gauge on $A \otimes_{K}{ }^{\iota} A$.

Proof. (1) The statement is obvious if $\sigma$ is of the first kind, so we assume that $\sigma$ is of the second kind. If there are two extensions of $v$ to $K$ then, by the fundamental inequality of valuation theory, $\Gamma_{v^{\prime}}=\Gamma_{v}$ and $K_{v^{\prime}}=F_{v}$. Let $P \in \operatorname{Lift}(\mathscr{Q})$. Then $P=P_{(\eta, Q)}$ as described in Theorem 8.1 and, again by Theorem 8.1, the set

$$
\left\{u b^{2} \rho \in K \mid u \in R_{v^{\prime}} \backslash I_{v^{\prime}}, b \in K, \rho \in \Omega_{\text {prod }} \text { such that } \pi_{v^{\prime}}(u) \cdot \eta(\rho) \in Q\right\}
$$

defines an ordering on $K$ extending $P$, which is not possible by Lemma 8.4 .

(2) This follows from (1) by [7, Prop. 2.1].

(3) $w \otimes w$ is a $v^{\prime}$-gauge on $A \otimes_{K}{ }^{\iota} A$ by [22, Cor. 1.28] and Remark 3.7, and is also a $v$-gauge by [7, Prop. 2.1]. It is clearly $\sigma \otimes{ }^{\iota} \sigma$-invariant.

In order to keep notation simple,we identify $A \otimes_{K}{ }^{\iota} A$ and $M_{m}(K)$ and consider the gauge $w \otimes w$ as being defined on $M_{m}(K)$.

We now write the form $T_{(A, \sigma)}$ as

$$
T_{(A, \sigma)} \simeq \rho_{1} h_{1} \perp \cdots \perp \rho_{k} h_{k},
$$

where $\rho_{1}, \ldots, \rho_{k}$ are distinct elements of $\Omega_{\text {prod }}$, and $h_{1}, \ldots, h_{k}$ are diagonal forms over $(K, \iota)$ with coefficients in $R_{v} \backslash I_{v}$. Since $m$ is represented by $T_{(A, \sigma)}$ we can assume that $\rho_{1}=1$, and that the first coefficient of $h_{1}$ is $m$.

Lemma 8.6. Assume that $\operatorname{Lift}(\mathscr{Q}) \neq \varnothing$. For every $i=1, \ldots, k$, either all coefficients of $h_{i}$ project via $\pi_{v}$ into $Q$ (in this case we define $\varepsilon_{i}:=1$ ), or all coefficients of $h_{i}$ project via $\pi_{v}$ into $-Q$ (in this case we define $\left.\varepsilon_{i}:=-1\right)$. 
Proof. Let $P \in \operatorname{Lift}(\mathscr{Q})$. By Theorem 8.2 , the form $T_{(A, \sigma)}$ is definite at $P$. By [3. Prop. 4.8 with $u=1], \sigma$ and thus $\sigma \otimes{ }^{\iota} \sigma$ are positive at $P$. Therefore, the involution $\operatorname{ad}_{T_{(A, \sigma)}}$ is positive at $P$, and by [4, Cor. 7.7] there is a positive cone $\mathscr{S}$ on $\left(M_{m}(K), \operatorname{ad}_{T_{(A, \sigma)}}\right)$ that contains 1 . We can therefore define the $\operatorname{ad}_{T_{(A, \sigma)} \text {-special }}$ $v$-gauge $w_{k, \mathscr{S}}$ on $M_{m}(K)$ (cf. Section 5.2 ; actually, since $w \otimes w$ is an $\operatorname{ad}_{T_{(A, \sigma)}}$ invariant $v$-gauge, we have $w \otimes w=w_{k, \mathscr{S}}$ by Theorem 5.16), and this $v$-gauge induces a positive cone $\mathscr{S}_{0}$ on $\left(\left(M_{m}(K)\right)_{0},\left(\operatorname{ad}_{T_{(A, \sigma)}}\right)_{0}\right)$ over $Q$ that contains 1 by Theorem 6.11 (or by property (C5)).

We consider the residue algebra with involution $\left(\left(M_{m}(K)\right)_{0},\left(\operatorname{ad}_{T_{(A, \sigma)}}\right)_{0}\right)$ with respect to the gauge $w_{k, \mathscr{S}}$. By Proposition 5.19, $\left(M_{m}(K)\right)_{0} \cong B_{1} \times \cdots \times B_{k}$, with each $B_{i}$ simple, and the residue involution is $\left(\operatorname{ad}_{T_{(A, \sigma)}}\right)_{0} \cong \operatorname{ad}_{\widetilde{h}_{1}} \times \cdots \times \operatorname{ad}_{\widetilde{h}_{k}}$. By Corollary 4.16, $\mathscr{S}_{0}=\mathscr{S}_{1} \times \cdots \times \mathscr{S}_{k}$ with $\mathscr{S}_{i}$ a positive cone on $\left(B_{i}, \operatorname{ad}_{\tilde{h}_{i}}\right)$ over $Q$, that contains 1. Therefore, the involution $\operatorname{ad}_{\tilde{h}_{i}}$ is positive at $Q$ (see [4, Cor. 7.7]). By [3, Prop. 4.4(iv)] (using the diagonal matrix $u$ that contains the coefficients of $\widetilde{h}_{i}$, and noting that $\left.\operatorname{ad}_{\widetilde{h}_{i}}=\left(\sigma_{0}^{t}\right)_{u}\right)$, the coefficients of $\widetilde{h}_{i}$ are either all in $Q$, or all in $-Q$.

Observe that $\varepsilon_{1}=1$ since the first coefficient of $h_{1}$ is $m$.

Proposition 8.7. Assume that $\operatorname{Lift}(\mathscr{Q}) \neq \varnothing$.

(1) $\operatorname{Lift}(\mathscr{Q})$ is the intersection of the Harrison set $H\left(\varepsilon_{1} \rho_{1}, \ldots, \varepsilon_{k} \rho_{k}\right)$ with the set of all liftings of $Q$ to $F$.

(2) $\Gamma_{w} \subseteq \Gamma_{w \otimes w}=\sum_{i, j=1}^{k} \frac{1}{2}\left(v\left(\rho_{i}\right)-v\left(\rho_{j}\right)\right)+\Gamma_{v^{\prime}}$.

Proof. (1) is a direct consequence of Theorem $8.2(4)$ and the definition of the elements $\varepsilon_{i}$ in Lemma 8.6. Indeed, a lifting $P$ of $Q$ is in $\operatorname{Lift}(\mathscr{Q})$ if and only if the coefficients of $T_{(A, \sigma)}$ are all in $P$ or all in $-P$, if and only if each $\varepsilon_{i} \rho_{i}$ is positive at $P$ (or negative at $P$, but this case is not possible since $\rho_{1}=1$ and $\varepsilon_{1}=1$ ).

(2) Let $P \in \operatorname{Lift}(\mathscr{Q})$, then $T_{(A, \sigma)}$ is positive at $P$ by Theorem 8.2 . It follows, using [4, Cor. 7.7] and as already observed in the proof of Lemma 8.6, that there exists a positive cone $\mathscr{S}$ on $\left(M_{m}(K), \operatorname{ad}_{T_{(A, \sigma)}}\right)$ over $P$ that contains 1 . We are therefore in the situation of Section 5.3 with $(E,-)=(K, \iota)$ and $h=T_{(A, \sigma)}$, and so may consider the $\operatorname{ad}_{T_{(A, \sigma)}}$-special $v$-gauge $w_{k, \mathscr{S}}$. By Proposition 3.8 and Theorem 5.16, this is the unique $\operatorname{ad}_{T_{(A, \sigma)}}$-invariant $v$-gauge on $\operatorname{End}_{K}(A)$. Therefore, $w \otimes w=w_{k, \mathscr{S}}$ and $\Gamma_{w \otimes w}=\Gamma_{w_{k, \mathscr{S}}}$. The result then follows by Proposition 5.19, using that $\Gamma_{w} \subseteq \Gamma_{w \otimes w}$ (see [22, Thm. 3.21(ii)]).

Lemma 8.8. Assume that $\sigma$ is of the second kind, and that for every lifting $P$ of $Q$ to $F$, the involution $\sigma$ is positive at $P$. Then $\Gamma_{v}=\Gamma_{v^{\prime}}$.

Proof. (This elegant argument was suggested to us by A. Wadsworth.) Write $K=F(\sqrt{d})$ for some $d \in F$. An ordering on $F$ extends to $K$ if and only if $d$ is positive at this ordering. By the assumption and Lemma 8.4, $d$ must be negative at every lifting of $Q$ to $F$. However, if $v(d)$ were not in $2 \Gamma_{v}$, then $d$ would be 
positive at some liftings of $Q$ by the Baer-Krull theorem. Hence, $v(d)$ must be an element of $2 \Gamma_{v}$, which implies that $\Gamma_{v^{\prime}}=\Gamma_{v}$.

We can now describe when every lifting of $Q$ to $P$ gives rise to a lifting of the positive cone $\mathscr{Q}$ that contains 1 .

Theorem 8.9. The following are equivalent:

(1) For every lifting $P$ of $Q$, there is a lifting $\mathscr{P}$ of $\mathscr{Q}$ that contains 1.

(2) $\Gamma_{w}=\Gamma_{v}$.

If one of these holds, then the number of liftings of $\mathscr{Q}$ to $(A, \sigma)$ that contain 1 is equal to $\left|\Gamma_{v} / 2 \Gamma_{v}\right|$.

Proof. (1) $\Rightarrow(2)$ : By Proposition 8.7(1) we obtain that the set of all liftings of $Q$ to $F$ is included in $H\left(\varepsilon_{1} \rho_{1}, \ldots, \varepsilon_{k} \rho_{k}\right)=H\left(1, \varepsilon_{2} \rho_{2} \ldots, \varepsilon_{k} \rho_{k}\right)$. By the Baer-Krull theorem, this can only happen if $k=1$ (since otherwise there would be a lifting of $P$ at which $\varepsilon_{2} \rho_{2}$ would be negative). In this case, Proposition 8.7(2) gives $\Gamma_{w}=\Gamma_{v^{\prime}}$. This concludes the proof if $\sigma$ is of the first kind. If $\sigma$ is of the second kind, Lemma 8.8 (using Theorem 8.2) gives $\Gamma_{v^{\prime}}=\Gamma_{v}$.

$(2) \Rightarrow(1)$ : Let $P$ be a lifting of $Q$ to $F$, determined by the function $\eta$, cf. Theorem 8.1. We define

$\mathscr{P}_{(\eta, Q)}:=\left\{\sum_{i=1}^{r} u_{i} \rho_{i} \sigma\left(x_{i}\right) x_{i} \mid r \in \mathbb{N}, u_{i} \in R_{v}^{\times}, \rho_{i} \in \Omega_{\mathrm{prod}}, \pi_{v}\left(u_{i}\right) \cdot \eta\left(\rho_{i}\right) \in Q, x_{i} \in A\right\}$

and observe that $u_{i} \rho_{i} \in P_{(\eta, Q)}$ and $1 \in \mathscr{P}_{(\eta, Q)}$. The set $\mathscr{P}$ is the closure of $\{1\}$ under the operations defining a positive cone over $P$, cf. properties (P2), (P3), and $(\mathrm{P} 4)$ in Definition 4.1. We first check that $\mathscr{P}_{(\eta, Q)}$ is a prepositive cone on $(A, \sigma)$ over $P_{(\eta, Q)}$ :

Properties (P1), (P2) and (P3) from Definition 4.1 are clearly satisfied. Property (P5) follows from Proposition 7.8 since the elements $u_{i} \rho_{i}$ are in $P_{(\eta, Q)} \backslash\{0\}$.

For (P4), and using that (P5) holds, it suffices to show that $P_{(\eta, Q)} \subseteq\left(\mathscr{P}_{(\eta, Q)}\right)_{F}$, cf. Remark 4.2. Thus, let $a \in P_{(\eta, Q)}$ and write $a=u b^{2} \rho$ with $v(u)=0, b \in F$, $\rho \in \Omega_{\text {prod }}$ and $\pi_{v}(u) \eta(\rho) \in Q$. Then, following the definition of $\mathscr{P}_{(\eta, Q)}$,

$$
a\left(u_{i} \rho_{i} \sigma\left(x_{i}\right) x_{i}\right)=\left(u u_{i}\right)\left(\rho \rho_{i}\right) \sigma\left(b x_{i}\right) b x_{i}
$$

is again an element of $\mathscr{P}_{(\eta, Q)}$.

Let $\mathscr{P}$ be the unique positive cone on $(A, \sigma)$ containing $\mathscr{P}_{(\eta, Q)}$. By Theorem 5.16 and Proposition [3.8, $w_{k, \mathscr{P}}$ is the unique $\sigma$-invariant $v$-gauge on $(A, \sigma)$ and $w=w_{k, \mathscr{P}}$. Therefore, $w$ and $\mathscr{P}$ are compatible by Proposition 6.5, and we conclude with Theorem 6.11 and Lemma 4.18.

This completes the proof of the equivalences. The final statement of the theorem is now clear: In this case, a lifting of $\mathscr{Q}$ that contains 1 is completely determined by the lifting $P$ of $Q$ (cf. Lemma 4.18), and the number of such liftings is determined by the Baer-Krull theorem. 
One might wonder if there could be a clear relation between the number of cosets of $\Gamma_{v}$ in $\Gamma_{w}$ and the number of liftings of $\mathscr{Q}$. This does not appear to be the case, as the following example shows:

Example 8.10. Let $F$ be a field and let $v$ be a real valuation on $F$ such that $\left\{\rho_{1}, \rho_{2}, \rho_{3}, \rho_{4}\right\} \subseteq \Gamma_{v}$ is a basis of $\Gamma_{v} / 2 \Gamma_{v}$. Let $A=M_{6}(F)$ and consider the involutions $\operatorname{ad}_{\varphi}$ and $\operatorname{ad}_{\psi}$ on $A$, where

$$
\varphi=\left\langle 1, \rho_{1}, \rho_{2}, \rho_{3}, \rho_{4}, \rho_{1} \rho_{2} \rho_{3} \rho_{4}\right\rangle \quad \text { and } \quad \psi=\left\langle 1, \rho_{1}, \rho_{2}, \rho_{3}, \rho_{1} \rho_{2}, \rho_{3} \rho_{4}\right\rangle .
$$

Let $P$ be an ordering on $F$ such that $\rho_{1}, \rho_{2}, \rho_{3}, \rho_{4} \in P$. Since $\operatorname{ad}_{\varphi}$ and $\operatorname{ad}_{\psi}$ are positive at $P$, there is a positive cone $\mathscr{P}_{1}$ on $\left(A, \operatorname{ad}_{\varphi}\right)$ over $P$ and a positive cone $\mathscr{P}_{2}$ on $\left(A, \mathrm{ad}_{\psi}\right)$ over $P$ such that both contain 1 , cf. [4, Cor. 7.7]. Thus, we may consider the $v$-gauges $w_{\mathbb{Q}, \mathscr{P}_{1}}$ and $w_{\mathbb{Q}, \mathscr{P}_{2}}$. Using Proposition 5.19 a direct, but lengthy, verification gives

$$
\left[\Gamma_{w_{\mathbb{Q}, \mathscr{P}}}: \Gamma_{v}\right]=16 \quad \text { and } \quad\left[\Gamma_{w_{\mathbb{Q}, \mathscr{P}_{2}}}: \Gamma_{v}\right]=13
$$

By Theorem 6.11, the positive cones $\mathscr{P}_{1}$ and $\mathscr{P}_{2}$ induce positive cones $\mathscr{Q}_{1}$ and $\mathscr{Q}_{2}$, respectively, on the residue algebras with involution of $\left(A, \operatorname{ad}_{\varphi}\right)$ with respect to $w_{\mathbb{Q}, \mathscr{P}_{1}}$ and $\left(A, \operatorname{ad}_{\psi}\right)$ with respect to $w_{\mathbb{Q}, \mathscr{P}_{2}}$, respectively. In fact,

$$
\left(A_{0},\left(\operatorname{ad}_{\varphi}\right)_{0}\right) \cong\left(A_{0},\left(\operatorname{ad}_{\psi}\right)_{0}\right) \cong\left(F_{v} \times F_{v} \times F_{v} \times F_{v} \times F_{v} \times F_{v}, \text { id }\right)
$$

by Proposition 5.19 and it follows from Corollary 4.16 that

$$
\mathscr{Q}_{1}=\mathscr{Q}_{2}=Q \times Q \times Q \times Q \times Q \times Q,
$$

where $Q$ is the ordering on $F_{v}$ induced by $P$.

Observe that $P$ is the only ordering on $F$ for which $\operatorname{ad}_{\varphi}$ is positive. Likewise, $P$ is the only ordering on $F$ for which $\operatorname{ad}_{\psi}$ is positive. Therefore,

$$
\left|\operatorname{Lift}\left(\mathscr{Q}_{1}\right)\right|=\left|\operatorname{Lift}\left(\mathscr{Q}_{2}\right)\right|=1 \text {, }
$$

by Theorem 8.2 ,

\section{Appendix A. Quaternionic matrices And Eigenvalues}

Given an ordering $P$ on $F$, several of our proofs consist of a reduction to the case of matrices over $F_{P}, F_{P}(\sqrt{-1})$ and $(-1,-1)_{F_{P}}$. The results that we present below in the case of $(-1,-1)_{F_{P}}$ are obtained when $F_{P}=\mathbb{R}$ using algebraic arguments, and are therefore valid in the case of a general real closed field instead of $\mathbb{R}$.

Let $R$ be a real closed field, $C:=R(\sqrt{-1})$ and $H:=(-1,-1)_{R}$, and let denote $\operatorname{id}_{R}$, complex conjugation or quaternion conjugation, respectively.

The theory of eigenvalues of real (symmetric) and complex (hermitian) matrices is well-known. Quaternionic matrices have also been studied extensively, see for example [26], from which we recall the following results.

Let $i$ and $j$ denote the generators of the quaternion division algebra $H$ over $R$. Let $M=M_{1}+M_{2} j \in M_{n}(H)$ with $M_{1}, M_{2} \in M_{n}(C)$. 
A quaternion $\lambda \in H$ is a left (resp. right) eigenvalue of $M$ if and only if $M x=\lambda x($ resp. $M x=x \lambda)$, for some $x \in H^{n} \backslash\{0\}$. Note that $M$ is invertible if and only if all its left and right eigenvalues are nonzero, cf. [26, Thm. 4.3]. An interesting property of the set of right eigenvalues of a quaternion matrix is that it is closed under conjugation: If $M x=x \lambda$ as above and $c \in H \backslash\{0\}$, then $M(x c)=(x c)\left(c^{-1} \lambda c\right)$.

We denote by $\chi_{M}$ the matrix

$$
\left(\begin{array}{cc}
M_{1} & M_{2} \\
-\overline{M_{2}} & \overline{M_{1}}
\end{array}\right) \in M_{2 n}(C)
$$

and consider the "characteristic polynomial" $p_{M}(X):=\operatorname{det}\left(X \cdot I_{2 n}-\chi_{M}\right) \in C[X]$. The proof of [26, Thm. 8.1(5)] shows that $p_{M}(X)$ belongs to $R[X]$. In fact, the map that sends $M$ to $\chi_{M}$ is a morphism from $M_{n}(H)$ to its splitting $M_{n}(H) \otimes_{R} C \cong$ $M_{2 n}(C)$. Therefore, $p_{M}$ is the reduced characteristic polynomial of $M$, and so has coefficients in $R$.

By [26, Thm. 8.1(5)] the Cayley-Hamilton theorem holds for $M$, i.e., $p_{M}(M)=$ 0 , and, for a quaternion $\lambda \in H, p_{M}(\lambda)=0$ if and only if $\lambda$ is a right eigenvalue of $M$.

Remark A.1. Let $M, N \in M_{n}(H)$. Since the map $M \mapsto \chi_{M}$ is a morphism, we have $\chi_{M N}=\chi_{M} \chi_{N}$ and thus $p_{M N}$ is the characteristic polynomial of $\chi_{M} \chi_{N}$, which is equal to the characteristic polynomial of $\chi_{N} \chi_{M}=\chi_{N M}$. Therefore $p_{M N}=p_{N M}$, and in particular the right eigenvalues of $M N$ are exactly the right eigenvalues of $N M$.

This can also be obtained by a direct computation, as well as the fact that, in case $M$ is invertible, the right eigenvalues of $M^{-1}$ are exactly the inverses of the right eigenvalues of $M$.

The Principal Axis Theorem holds for quaternion matrices by [26, Cor. 6.2]: if $M \in M_{n}(H)$ is such that $\bar{M}^{t}=M$, then there exist a unitary matrix $U \in M_{n}(H)$ (i.e., $\bar{U}^{t}=U^{-1}$ ) and scalars $\lambda_{1}, \ldots, \lambda_{n} \in R$ such that $\bar{U}^{t} M U=\operatorname{diag}\left(\lambda_{1}, \ldots, \lambda_{n}\right)$.

A matrix $M \in M_{n}(H)$ such that $\bar{M}^{t}=M$ is called positive semidefinite if $\bar{x}^{t} M x \geqslant 0$ for all $x \in H^{n}$, cf. [26, Rem. 6.1]. Negative semidefinite matrices are defined similarly.

Lemma A.2. Let $M \in M_{n}(H)$. Then

(1) If $\bar{M}^{t}=M$ and $\lambda \in H$ is a right eigenvalue of $M$, then $\lambda \in R$.

(2) If $\bar{M}^{t}=M$, then $M$ is positive semidefinite if and only if $M$ only has nonnegative right eigenvalues.

(3) If $U \in M_{n}(H)$ is invertible, then the right eigenvalues of $M$ are precisely the right eigenvalues of $U^{-1} M U$. 
(4) If $\bar{M}^{t}=M$ and $\lambda \in H$, then $\lambda$ is a right eigenvalue of $M$ if and only if for every unitary $U \in M_{n}(H)$ such that $\bar{U}^{t} M U$ is diagonal, $\lambda$ is one of the diagonal elements.

(5) If $\bar{M}^{t}=M$, then $M$ is positive semidefinite if and only if whenever $U$ is unitary such that $\bar{U}^{t} M U$ is diagonal, its diagonal elements are nonnegative.

Proof. (1) Let $x \in H^{n} \backslash\{0\}$ be such that $M x=x \lambda$. Then $\lambda=\left(\bar{x}^{t} x\right)^{-1}\left(\bar{x}^{t} M x\right) \in R$.

(2) This is [26, Rem. 6.1].

(3) This is immediate.

(4) Let $\lambda \in H$ be a right eigenvalue of $M$. Observe that since $\bar{M}^{t}=M$, the standard argument shows that $\lambda \in R$. Let $\bar{U}^{t} M U=\operatorname{diag}\left(\lambda_{1}, \ldots, \lambda_{n}\right)$ for $\lambda_{1}, \ldots, \lambda_{n} \in R$. By (3) there exists $x \in H^{n} \backslash\{0\}$ such that $\bar{U}^{t} M U x=x \lambda$. If, for instance, $x_{1} \neq 0$, we obtain $\lambda_{1} x_{1}=x_{1} \lambda=\lambda x_{1}$. The result follows. Conversely, the diagonal elements of $\bar{U}^{t} M U$ are clearly right eigenvalues of $\bar{U}^{t} M U$, and the result follows from (3).

(5) This follows from (2) and (4).

\section{ACKNOWLEDGMENTS}

We would like to take this opportunity to extend a very warm thank you to Adrian Wadsworth, who read a previous version of this paper in great detail and discovered some serious errors. He also suggested some more elegant arguments and greatly helped our work in Section 8 via a stimulating exchange of emails. In particular, he drew our attention to the condition $\Gamma_{w}=\Gamma_{v}$ that led to Theorem 8.9 .

\section{REFERENCES}

[1] V. Astier and T. Unger. Signatures of hermitian forms and the Knebusch trace formula. Math. Ann., 358(3-4):925-947, 2014.

[2] V. Astier and T. Unger. Signatures of hermitian forms and "prime ideals" of Witt groups. Adv. Math., 285:497-514, 2015.

[3] V. Astier and T. Unger. Signatures of hermitian forms, positivity, and an answer to a question of Procesi and Schacher. J. Algebra, 508:339-363, 2018.

[4] V. Astier and T. Unger. Positive cones on algebras with involution. Adv. Math., 361:106954, 2020.

[5] J. Bochnak, M. Coste, and M.-F. Roy. Real algebraic geometry, volume 36 of Ergebnisse der Mathematik und ihrer Grenzgebiete (3). Springer-Verlag, Berlin, 1998.

[6] A.J. Engler and A. Prestel. Valued fields, Springer Monographs in Mathematics. SpringerVerlag, Berlin, 2005.

[7] M.A. Ferreira and A.R. Wadsworth, Value functions and Dubrovin valuation rings on simple algebras. Trans. Amer. Math. Soc., 368(3):1681-1734, 2016.

[8] P.A. Grillet. Abstract algebra. Second edition. Graduate Texts in Mathematics, 242. Springer, New York, 2007.

[9] S.S. Holland, Jr. *-valuations and ordered *-fields. Trans. Amer. Math. Soc., 262(1):219 243,1980 . 
[10] M. Knebusch and C. Scheiderer. Einführung in die reelle Algebra, volume 63 of Vieweg Studium: Aufbaukurs Mathematik. Friedr. Vieweg \& Sohn, Braunschweig, 1989.

[11] M.-A. Knus. Quadratic and Hermitian forms over rings, volume 294 of Grundlehren der Mathematischen Wissenschaften. Springer-Verlag, Berlin, 1991.

[12] M.-A. Knus, A. Merkurjev, M. Rost, and J.-P. Tignol. The book of involutions, volume 44 of American Mathematical Society Colloquium Publications. American Mathematical Society, Providence, RI, 1998.

[13] A. Kulshrestha. Strongly anisotropic involutions on central simple algebras. Comm. Algebra, 39(5):1686-1704, 2011.

[14] T.Y. Lam. Orderings, valuations and quadratic forms, volume 52 of CBMS Regional Conference Series in Mathematics. Amer. Math. Soc., Providence, RI, 1983.

[15] D.W. Larmour. A Springer theorem for Hermitian forms. Math. Z., 252(3):459-472, 2006.

[16] M. Marshall. Spaces of orderings and abstract real spectra. Lecture Notes in Mathematics, 1636. Springer-Verlag, Berlin, 1996.

[17] P. Morandi. The Henselization of a valued division algebra. J. Algebra, 122(1): 232-243, 1989.

[18] P. Morandi. Value functions on central simple algebras. Trans. Amer. Math. Soc., 315(2):605-622, 1989.

[19] A. Prestel. Lectures on formally real fields, volume 1093 of Lecture Notes in Mathematics. Springer-Verlag, Berlin, 1984.

[20] A. Prestel and C.N. Delzell. Positive polynomials. Springer Monographs in Mathematics. Springer-Verlag, Berlin, 2001. From Hilbert's 17th problem to real algebra.

[21] W. Scharlau. Quadratic and Hermitian forms, Grundlehren der Mathematischen Wissenschaften, 270. Springer-Verlag, Berlin, 1985.

[22] J.-P. Tignol and A.R. Wadsworth. Value functions and associated graded rings for semisimple algebras. Trans. Amer. Math. Soc., 362(2):687-726, 2010.

[23] J.-P. Tignol and A.R. Wadsworth. Valuations on algebras with involution. Math. Ann., 351(1):109-148, 2011.

[24] J.-P. Tignol and A.R. Wadsworth. Value functions on simple algebras, and associated graded rings. Springer Monographs in Mathematics. Springer, Cham, 2015.

[25] A.R. Wadsworth. Valuation theory on finite dimensional division algebras. In Valuation theory and its applications, Vol. I (Saskatoon, SK, 1999), volume 32 of Fields Inst. Commun., pages 385-449. Amer. Math. Soc., Providence, RI, 2002.

[26] F. Zhang. Quaternions and matrices of quaternions. Linear Algebra Appl., 251:21-57, 1997.

School of Mathematics and Statistics, University College Dublin, Belfield, Dublin 4, IRELAND

Email address: vincent.astier@ucd.ie, thomas.unger@ucd.ie 